\title{
The equivalence theorem for logarithmic interpolation spaces in the quasi-Banach case
}

\author{
Blanca F. Besoy and Fernando Cobos
}

\begin{abstract}
We study the description by means of the $J$-functional of logarithmic interpolation spaces $\left(A_{0}, A_{1}\right)_{1, q, \mathbb{A}}$ in the category of the $p$-normed quasi-Banach couples $(0<p \leq 1)$. When $\left(A_{0}, A_{1}\right)$ is a Banach couple, it is known that the description changes depending on the relationship between $q$ and $\mathbb{A}$. In our more general setting, the parameter $p$ also has an important role as the results show.
\end{abstract}

Keywords. Logarithmic interpolation spaces; $J$-functional; $K$-functional; characteristic function

Mathematics Subject Classification (2010). 46M35,46B70

\section{Introduction}

The real interpolation method $\left(A_{0}, A_{1}\right)_{\theta, q}$ is an useful tool to work in PDEs, Harmonic Analysis, Approximation Theory, Function Spaces and Operator Theory. See the books by Butzer and Berens [9], Bergh and Löfström [4], Triebel [32], König [25], Bennett and Sharpley [3] and Brudnyi and Krugljak [8]. The most common definition of the real method is given in terms of Peetre's $K$-functional. But there is an equivalent description using the $J$-functional, which is also very useful. For instance, to establish the reiteration theorem, to study duality problems or interpolation of compactness by the real method, the $J$-representation of the $K$-spaces $\left(A_{0}, A_{1}\right)_{\theta, q}$ plays a central role (see [4] and the papers by Cwikel and Peetre [17] and Cobos, Kühn and Schonbek [15]).

Logarithmic perturbations $\left(A_{0}, A_{1}\right)_{\theta, q, \mathbb{A}}$ of the real method are also very useful in applications. Here $0 \leq \theta \leq 1,0<q \leq \infty, \mathbb{A}=\left(\alpha_{0}, \alpha_{\infty}\right) \in \mathbb{R}^{2}$ and the

B.F. Besoy: Departamento de Análisis Matemático y Matemática Aplicada, Facultad de Matemáticas, Universidad Complutense de Madrid, Plaza de Ciencias 3, 28040 Madrid, Spain; blanca.f.besoy@ucm.es.

F. Cobos: Departamento de Análisis Matemático y Matemática Aplicada, Facultad de Matemáticas, Universidad Complutense de Madrid, Plaza de Ciencias 3, 28040 Madrid, Spain; cobos@mat.ucm.es (Corresponding author). 
space $\left(A_{0}, A_{1}\right)_{\theta, q, \mathbb{A}}$ is defined similarly to $\left(A_{0}, A_{1}\right)_{\theta, q}$ but inserting the weight

$$
\ell^{\mathbb{A}}(t)= \begin{cases}(1+|\log t|)^{\alpha_{0}} & \text { if } 0<t \leq 1 \\ (1+|\log t|)^{\alpha_{\infty}} & \text { if } 1<t<\infty\end{cases}
$$

with the $K$-functional. See the papers by Gustavsson [23], Doktorskii [18], Evans and Opic [21], Evans, Opic and Pick [22], Edmunds and Opic [20] and Cobos and Segurado [16]. For suitable choices of $\mathbb{A}$ we can allow that $\theta$ takes the extreme values 1 and 0 . Spaces $\left(A_{0}, A_{1}\right)_{j, q, \mathbb{A}}$ are very close to $A_{j}, j=0,1$. They are also connected with the so-called limiting interpolation spaces for ordered couples which have been studied by Cobos, Fernández-Cabrera, Kühn and Ullrich [12], Cobos and Kühn [14], Cobos and Domínguez [10] and Cobos, Domínguez and Triebel [11], among other authors.

For couples of Banach spaces the description of $\left(A_{0}, A_{1}\right)_{1, q, \mathbb{A}}$ in terms of the $J$-functional has been studied by Cobos and Segurado [16] in the case $1 \leq$ $q \leq \infty$ and by the present authors [5] when $0<q \leq 1$. It turns out that if $\alpha_{0}+1 / q<0<\alpha_{\infty}+1 / q$, to go from the $K$-representation of $\left(A_{0}, A_{1}\right)_{1, q, \mathbb{A}}$ to the $J$-representation, one should correct the exponents of the logarithm by adding 1 when $1 \leq q \leq \infty$, but if $0<q<1$ then one should add $1 / q$ and moreover the Gagliardo completions of $A_{0}$ and $A_{1}$ are involved. The extreme case $q=\infty$, $\alpha_{0}=0$ and $\alpha_{\infty}>0$ has been studied by Besoy, Cobos and Fernández-Cabrera [7]. Then the $J$-representation is of another nature.

When $0<q<1$ the space $\left(A_{0}, A_{1}\right)_{1, q, \mathbb{A}}$ is not Banach but quasi-Banach. In fact, see for example the books by Bergh and Löfström [4], Triebel [32] and König [25] or the paper by Nilsson [27], because of applications it is suitable to work with the real method defined for $p$-normed quasi-Banach couples $(0<p \leq$ $1)$. Accordingly, we consider here the logarithmic spaces $\left(A_{0}, A_{1}\right)_{1, q, \mathbb{A}}$ in that category of couples and investigate their description in terms of the $J$-functional.

Our results disclose that now the correction factor in the exponents of the logarithm depends on the relation among $p, q$ and $\mathbb{A}$. Sometimes, although $\left(A_{0}, A_{1}\right)_{1, q, \mathbb{A}}$ admits a description as a $J$-space $\left(A_{0}, A_{1}\right)_{\Gamma ; J}$, the sequence lattice $\Gamma$ is not a logarithmic sequence space of the kind $\ell_{q}\left(2^{-m} \ell^{\mathbb{M}}\left(2^{m}\right)\right)$ but a sequence of a different scale. In such cases we determine the best $\mathbb{M}$ and $\mathbb{B}$ such that

$$
\left(A_{0}, A_{1}\right)_{\ell_{q}\left(2^{-m} \ell^{\mathbb{B}}\left(2^{m}\right)\right) ; J} \hookrightarrow\left(A_{0}, A_{1}\right)_{1, q, \mathbb{A}} \hookrightarrow\left(A_{0}, A_{1}\right)_{\ell_{q}\left(2^{-m} \ell^{\mathbb{M}}\left(2^{m}\right)\right) ; J}
$$

We start by recalling the basic properties of $\left(A_{0}, A_{1}\right)_{1, q, \mathbb{A}}$ in Section 2, where we also determine its characteristic function. Logarithmic $J$-spaces are introduced and studied in Section 3. Finally, in Section 4, we establish the equivalence theorems and the embedding theorems. 


\section{Preliminaries}

Let $\left(A,\|\cdot\|_{A}\right)$ be a quasi-Banach space with constant $c_{A} \geq 1$ in the quasitriangle inequality. Let $0<p \leq 1$ such that $c_{A}=2^{1 / p-1}$. As it is shown in $[26, \S 15.10]$ or $[25$, Proposition 1.c.5], there is another quasi-norm $\||\cdot|\|$ on $A$ which is equivalent to $\|\cdot\|_{A}$ and such that \|\|$\cdot \|\left.\right|^{p}$ satisfies the triangle inequality. We say that $(A,\||\cdot|\|)$ is a $p$-normed quasi-Banach space. Note that if $0<r<p$ then $(A,\||\cdot|\| \mid)$ is also an $r$-normed space. Conversely, if $(A, \||\cdot|||)$ is a $p$-normed space then it is quasi-normed with constant $2^{1 / p-1}$.

Let $\mathcal{A}$ be a Hausdorff topological vector space and let $A_{j}, j=0,1$, be ( $p$ normed) quasi-Banach spaces such that $A_{j} \hookrightarrow \mathcal{A}$, where $\hookrightarrow$ means continuous embedding. Then we say that $\bar{A}=\left(A_{0}, A_{1}\right)$ is a (p-normed) quasi-Banach couple.

For $t>0$ and $a \in A_{0}+A_{1}$, Peetre's $K$-functional is defined by

$$
K(t, a)=K\left(t, a ; A_{0}, A_{1}\right)=\inf \left\{\left\|a_{0}\right\|_{A_{0}}+t\left\|a_{1}\right\|_{A_{1}}: a=a_{0}+a_{1}, a_{j} \in A_{j}\right\} .
$$

It is useful to consider also

$$
K_{p}(t, a)=K_{p}\left(t, a ; A_{0}, A_{1}\right)=\inf \left\{\left(\left\|a_{0}\right\|_{A_{0}}^{p}+t^{p}\left\|a_{1}\right\|_{A_{1}}^{p}\right)^{1 / p}: a=a_{0}+a_{1}, a_{j} \in A_{j}\right\} .
$$

Peetre's J-functional is given by

$$
J(t, a)=J\left(t, a ; A_{0}, A_{1}\right)=\max \left\{\|a\|_{A_{0}}, t\|a\|_{A_{1}}\right\}, a \in A_{0} \cap A_{1} .
$$

The functionals $K(t, \cdot)$ and $K_{p}(t, \cdot)$ are quasi-norms in $A_{0}+A_{1}$ and $J(t, \cdot)$ is a quasi-norm in $A_{0} \cap A_{1}$. We can take the same constant $c \geq 1$ in the quasitriangle inequality for any $t>0$. Moreover, $K(1, \cdot), J(1, \cdot)$ coincide with the usual quasi-norms $\|\cdot\|_{A_{0}+A_{1}},\|\cdot\|_{A_{0} \cap A_{1}}$ of $A_{0}+A_{1}$ and $A_{0} \cap A_{1}$, respectively. Note also that $K(t, \cdot)$ and $K_{p}(t, \cdot)$ are equivalent quasi-norms

$$
K(t, a) \leq K_{p}(t, a) \leq 2^{1 / p-1} K(t, a), a \in A_{0}+A_{1} .
$$

Another useful property is that if $\|\cdot\|_{A_{j}}$ is a $p$-norm for $j=0,1$, then $K_{p}(t, \cdot)$ and $J(t, \cdot)$ are $p$-norms.

Let $0 \leq \theta \leq 1,0<q \leq \infty$ and $\mathbb{A}=\left(\alpha_{0}, \alpha_{\infty}\right) \in \mathbb{R}^{2}$. The logarithmic interpolation space $\bar{A}_{\theta, q, \mathbb{A}}=\left(A_{0}, A_{1}\right)_{\theta, q, \mathbb{A}}$ consists of all those $a \in A_{0}+A_{1}$ which have a finite quasi-norm

$$
\|a\|_{\bar{A}_{\theta, q, \mathbb{A}}}=\|a\|_{\left(A_{0}, A_{1}\right)_{\theta, q, \mathbb{A}}}=\left(\sum_{m=-\infty}^{\infty}\left[2^{-\theta m} \ell^{\mathbb{A}}\left(2^{m}\right) K\left(2^{m}, a\right)\right]^{q}\right)^{1 / q}
$$

(the sum should be replaced by the supremum when $q=\infty$ ). Here $\ell(t)=$ $1+|\log t|$ and

$$
\ell^{\mathbb{A}}(t)=\ell^{\left(\alpha_{0}, \alpha_{\infty}\right)}(t)= \begin{cases}\ell^{\alpha_{0}}(t) & \text { if } 0<t \leq 1 \\ \ell^{\alpha_{\infty}}(t) & \text { if } 1 \leq t<\infty .\end{cases}
$$


See $[5,16,21,22]$. When $\mathbb{A}=(0,0)$ and $0<\theta<1$ then $\left(A_{0}, A_{1}\right)_{\theta, q,(0,0)}=$ $\left(A_{0}, A_{1}\right)_{\theta, q}$ is the real interpolation space (see $\left.[3,4,8,9,32]\right)$. For $\mathbb{A} \neq(0,0)$ and $0<\theta<1$ the space $\left(A_{0}, A_{1}\right)_{\theta, q, \mathbb{A}}$ is a special case of the real method with a function parameter (see $[23,24,31]$ ). Note also that the equality

$$
K\left(t, a ; A_{0}, A_{1}\right)=t K\left(t^{-1}, a ; A_{1}, A_{0}\right), a \in A_{0}+A_{1},
$$

implies that $\left(A_{0}, A_{1}\right)_{0, q,\left(\alpha_{0}, \alpha_{\infty}\right)}=\left(A_{1}, A_{0}\right)_{1, q,\left(\alpha_{\infty}, \alpha_{0}\right)}$.

Subsequently, we are interested in the spaces that arise when $\theta=1$. On the contrary to the case of the real method where $A_{0} \cap A_{1} \hookrightarrow\left(A_{0}, A_{1}\right)_{\theta, q}$, now it may happen that $\left(A_{0}, A_{1}\right)_{1, q, \mathbb{A}}=\{0\}$. To avoid it, we assume that

$$
\begin{cases}\alpha_{0}+1 / q<0 & \text { if } 0<q<\infty \\ \alpha_{0} \leq 0 & \text { if } q=\infty\end{cases}
$$

(see [22, Theorem 2.2]). Under this assumption, the space $\left(A_{0}, A_{1}\right)_{1, q, \mathbb{A}}$ is an intermediate space with respect to $\bar{A}$ in the sense that the following embeddings hold

$$
A_{0} \cap A_{1} \hookrightarrow\left(A_{0}, A_{1}\right)_{1, q, \mathbb{A}} \hookrightarrow A_{0}+A_{1} .
$$

Moreover, if $\bar{B}=\left(B_{0}, B_{1}\right)$ is another quasi-Banach couple and the operator $T \in \mathcal{L}\left(A_{0}+A_{1}, B_{0}+B_{1}\right)$ is a linear operator such that $T: A_{j} \rightarrow B_{j}$ is bounded for $j=0,1$, then the restriction $T: \bar{A}_{1, q, \mathbb{A}} \rightarrow \bar{B}_{1, q, \mathbb{A}}$ is also bounded. So, in the terminology of $[4,32]$, the logarithmic method with parameters $1, q, \mathbb{A}$ is an interpolation functor in the category of all quasi-Banach couples.

If $A$ is a quasi-Banach space intermediate with respect to $\bar{A}$ then we write $A^{\circ}$ for the closure of $A_{0} \cap A_{1}$ in $A$.

It follows from the fundamental lemma (see [4, Lemma 3.3.2]) that $a \in$ $\left(A_{0}+A_{1}\right)^{\circ}$ if and only if

$$
\min (1,1 / t) K(t, a) \rightarrow 0 \text { as } t \rightarrow 0 \text { and as } t \rightarrow \infty .
$$

Assume that, in addition to (2.1), we have

$$
\begin{cases}\alpha_{\infty}+1 / q \geq 0 & \text { if } 0<q<\infty \\ \alpha_{\infty}>0 & \text { if } q=\infty\end{cases}
$$

Then, given any $a \in\left(A_{0}, A_{1}\right)_{1, q, \mathbb{A}}$, we have

$$
\left(\sum_{m=-\infty}^{\infty}\left[2^{-m} \ell^{\mathbb{A}}\left(2^{m}\right) K\left(2^{m}, a\right)\right]^{q}\right)^{1 / q}<\infty \quad \text { but } \quad \sum_{m=1}^{\infty} \ell^{\alpha_{\infty} q}\left(2^{m}\right)=\infty
$$

and $\sum_{m=-\infty}^{0}\left[2^{-m} \ell^{\alpha_{0}}\left(2^{m}\right)\right]^{q}=\infty$. Therefore, $\min (1,1 / t) K(t, a) \rightarrow 0$ as $t \rightarrow 0$ and as $t \rightarrow \infty$. Whence

$$
\left(A_{0}, A_{1}\right)_{1, q, \mathbb{A}} \subseteq\left(A_{0}+A_{1}\right)^{\circ} .
$$


Remark 2.1. Note that if (2.2) does not hold then (2.3) may fail. An example can be found in [5, Example 2.1].

Subsequently, if $X$ and $Y$ are quantities depending on certain parameters some of them being the significant parameters in our reasoning, we write $X \lesssim Y$ if $X \leq c Y$ with a constant $c>0$ independent of the significant parameters. We put $X \sim Y$ if $X \lesssim Y$ and $Y \lesssim X$.

In order to give an example let $(\Omega, \mu)$ be a $\sigma$-finite measure space and let $0<r<\infty$. According to [4, Theorem 5.2.1] we have that

$$
K\left(t, f ; L_{r}(\Omega), L_{\infty}(\Omega)\right)=\left(\int_{0}^{t^{r}}\left(f^{*}(s)\right)^{r} d s\right)^{1 / r}
$$

where $f^{*}$ is the non-increasing rearrangement of $f$. Let $0<q \leq \infty$ and $\mathbb{A}=$ $\left(\alpha_{0}, \alpha_{\infty}\right) \in \mathbb{R}^{2}$ satisfying (2.1). By a change of variable and using Hardy's inequality we obtain

$$
\begin{aligned}
\|f\|_{\left(L_{r}(\Omega), L_{\infty}(\Omega)\right)_{1, q, \mathbb{A}}} & \sim\left(\int_{0}^{\infty}\left[t^{-1} \ell^{\mathbb{A}}(t)\left(\int_{0}^{t^{r}}\left(f^{*}(s)\right)^{r} d s\right)^{1 / r}\right]^{q} \frac{d t}{t}\right)^{1 / q} \\
& \sim\left(\int_{0}^{\infty}\left[u^{-1 / r} \ell^{\mathbb{A}}(u)\left(\int_{0}^{u}\left(f^{*}(s)\right)^{r} d s\right)^{1 / r}\right]^{q} \frac{d u}{u}\right)^{1 / q} \\
& \sim\left(\int_{0}^{\infty}\left[\ell^{\mathbb{A}}(u) f^{*}(u)\right]^{q} \frac{d u}{u}\right)^{1 / q} .
\end{aligned}
$$

This yields that $\left(L_{r}(\Omega), L_{\infty}(\Omega)\right)_{1, q, \mathbb{A}}$ coincides, with equivalence of quasi-norms, with the generalized Lorentz-Zygmund space $L_{\infty, q ; \mathbb{A}}(\Omega)$. We refer to $[19,28]$ for properties of these function spaces.

If $\left(A,\|\cdot\|_{A}\right)$ is a quasi-Banach space and $s>0$, we write $s A$ for the space $A$ endowed with the quasi-norm $s\|\cdot\|_{A}$.

The characteristic function $\Phi_{\mathcal{F}}$ of an interpolation functor $\mathcal{F}$ is the function defined by

$$
\mathcal{F}(\mathbb{R},(1 / t) \mathbb{R})=\left(1 / \Phi_{\mathcal{F}}(t)\right) \mathbb{R}, t>0
$$

(see $[8,24,29])$. Next we describe the characteristic function $\Phi_{q, \mathbb{A}}$ of $(\cdot, \cdot)_{1, q, \mathbb{A}}$.

In what follows, we put $\ell \ell(t)=1+\log (1+|\log t|)$ and define $\ell \ell^{\mathbb{A}}(t)$ similarly to $\ell^{\mathbb{A}}(t)$.

If $\mathbb{A}=\left(\alpha_{0}, \alpha_{\infty}\right) \in \mathbb{R}^{2}$ and $\lambda \in \mathbb{R}$, we write $\mathbb{A}+\lambda=\left(\alpha_{0}+\lambda, \alpha_{\infty}+\lambda\right)$ and $\lambda \mathbb{A}=\left(\lambda \alpha_{0}, \lambda \alpha_{\infty}\right)$.

The following auxiliary result is useful.

Lemma 2.2. Let $\mathbb{A}=\left(\alpha_{0}, \alpha_{\infty}\right) \in \mathbb{R}^{2}$ and $0<q \leq \infty$ satisfying (2.1). Put

$$
\mathrm{v}_{q, \mathbb{A}}\left(2^{k}\right)=\left(\sum_{m=-\infty}^{\infty}\left[\min \left(1,2^{m-k}\right) 2^{-m} \ell^{\mathbb{A}}\left(2^{m}\right)\right]^{q}\right)^{1 / q}, k \in \mathbb{Z} .
$$


Then we have for $0<q<\infty$

$$
\mathrm{v}_{q, \mathbb{A}}\left(2^{k}\right) \sim \begin{cases}2^{-k} \ell^{\mathbb{A}+1 / q}\left(2^{k}\right) & \text { if } \alpha_{\infty}+1 / q>0 \\ 2^{-k} \ell^{\mathbb{A}+1 / q}\left(2^{k}\right) \ell \ell^{(0,1 / q)}\left(2^{k}\right) & \text { if } \alpha_{\infty}+1 / q=0 \\ 2^{-k} \ell^{\left(\alpha_{0}+1 / q, 0\right)}\left(2^{k}\right) & \text { if } \alpha_{\infty}+1 / q<0\end{cases}
$$

and for $q=\infty$

$$
\mathrm{v}_{\infty, q}\left(2^{k}\right) \sim \begin{cases}2^{-k} \ell^{\mathbb{A}}\left(2^{k}\right) & \text { if } \alpha_{\infty} \geq 0 \\ 2^{-k} \ell^{\left(\alpha_{0}, 0\right)}\left(2^{k}\right) & \text { if } \alpha_{\infty}<0\end{cases}
$$

Proof. If $0<q<\infty$ then the result follows proceeding as in [5, Lemma 3.1]. Suppose $q=\infty$. We have

$$
\begin{aligned}
\mathrm{v}_{\infty, q}\left(2^{k}\right) & =\sup _{m \in \mathbb{Z}}\left\{\min \left(1,2^{m-k}\right) 2^{-m} \ell^{\mathbb{A}}\left(2^{m}\right)\right\} \\
& =\max \left\{\sup _{m \leq k}\left\{2^{-k} \ell^{\mathbb{A}}\left(2^{m}\right)\right\}, \sup _{m \geq k}\left\{2^{-m} \ell^{\mathbb{A}}\left(2^{m}\right)\right\}\right\} \\
& \sim 2^{-k} \sup _{m \leq k}\left\{\ell^{\mathbb{A}}\left(2^{m}\right)\right\} .
\end{aligned}
$$

If $k \leq 0$, since $\alpha_{0} \leq 0$, we get

$$
\mathrm{v}_{\infty, q}\left(2^{k}\right) \sim 2^{-k} \sup _{m \leq k}\left\{\left(1-\log 2^{m}\right)^{\alpha_{0}}\right\}=2^{-k} \ell^{\alpha_{0}}\left(2^{k}\right) .
$$

If $k>0$, we have

$$
\begin{aligned}
\mathrm{v}_{\infty, \mathbb{A}}\left(2^{k}\right) & \sim 2^{-k} \max \left(\sup _{m \leq 0}\left\{(1-\log t)^{\alpha_{0}}\right\}, \sup _{0 \leq m \leq k}\left\{(1+\log t)^{\alpha_{\infty}}\right\}\right) \\
& =2^{-k} \max \left(1, \sup _{0 \leq m \leq k}\left\{\left(1+\log 2^{m}\right)^{\alpha_{\infty}}\right\}\right) \\
& = \begin{cases}2^{-k} \ell^{\alpha_{\infty}}\left(2^{k}\right) & \text { if } \alpha_{\infty} \geq 0, \\
2^{-k} & \text { if } \alpha_{\infty}<0 .\end{cases}
\end{aligned}
$$

This completes the proof.

Proposition 2.3. Let $\left(A,\|\cdot\|_{A}\right)$ be a p-normed quasi-Banach space and let $\mathbb{A}=$ $\left(\alpha_{0}, \alpha_{\infty}\right) \in \mathbb{R}^{2}$ and $0<q \leq \infty$ satisfying (2.1). Then, with equivalence of quasi-norms, we have

$$
\left(A, 2^{-k} A\right)_{1, q, \mathbb{A}}=\mathrm{v}_{q, \mathbb{A}}\left(2^{k}\right) A,
$$

where the constants in the equivalence depend on $p$ but they are independent of $k \in \mathbb{Z}$ and of the concrete p-normed space $A$. 
Proof. Let $0<p \leq 1$ such that $A$ is p-normed. Take any $a \in A$ and $m \in \mathbb{Z}$. It is not hard to check that $K_{p}\left(2^{m}, a\right)=\min \left(1,2^{m-k}\right)\|a\|_{A}$. Therefore

$$
\begin{aligned}
\|a\|_{\left(A, 2^{-k} A\right)_{1, q, \mathbb{A}}} & \sim\left(\sum_{m=-\infty}^{\infty}\left[\min \left(1,2^{m-k}\right) 2^{-m} \ell^{\mathbb{A}}\left(2^{m}\right)\right]^{q}\right)^{1 / q}\|a\|_{A} \\
& =\mathrm{v}_{q, \mathbb{A}}\left(2^{k}\right)\|a\|_{A} .
\end{aligned}
$$

Corollary 2.4. Let $\mathbb{A}=\left(\alpha_{0}, \alpha_{\infty}\right) \in \mathbb{R}^{2}$ and $0<q \leq \infty$ satisfying (2.1). Then $\Phi_{q, \mathbb{A}}\left(2^{k}\right) \sim \mathrm{v}_{q, \mathbb{A}}\left(2^{k}\right)^{-1}$.

\section{J-spaces}

Next we recall the general real interpolation method realized by means of the $J$-functional. See the monographs by Peetre [30] and Brudnyi and Krugljak [8]. Since we are interested in quasi-Banach couples, following the paper by Nilsson [27], we work with the general $J$-method described in discrete way.

By a quasi-Banach sequence lattice $\left(\Gamma,\|\cdot\|_{\Gamma}\right)$ we mean a quasi-Banach space of real valued sequences with $\mathbb{Z}$ as index set and satisfying the following two properties:

i) $\Gamma$ contains all sequences with only finitely many non-zero coordinates.

ii) Whenever $\left|\xi_{m}\right| \leq\left|\eta_{m}\right|$ for each $m \in \mathbb{Z}$ and $\left(\eta_{m}\right) \in \Gamma$, then $\left(\xi_{m}\right) \in \Gamma$ and $\left\|\left(\xi_{m}\right)\right\|_{\Gamma} \leq\left\|\left(\eta_{m}\right)\right\|_{\Gamma}$.

For $0<q<\infty$, the space $\ell_{q}$ of $q$-summable sequences and the space $\ell_{\infty}$ of bounded sequences are examples of quasi-Banach sequence lattices. Another example is the space $\ell_{q}\left(2^{-m}\right)$, formed by all sequences $\left(\xi_{m}\right)$ such that $\left(2^{-m} \xi_{m}\right) \in$ $\ell_{q}$

Let $0<p \leq 1$. The quasi-Banach sequence lattice $\left(\Gamma,\|\cdot\|_{\Gamma}\right)$ is said to be $(p, J)$-non-trivial if $\Gamma \hookrightarrow \ell_{p}+\ell_{p}\left(2^{-m}\right)$, that is,

$$
\sup \left\{\left(\sum_{m=-\infty}^{\infty}\left[\min \left(1,2^{-m}\right)\left|\xi_{m}\right|\right]^{p}\right)^{1 / p}:\left\|\left(\xi_{m}\right)\right\|_{\Gamma} \leq 1\right\}<\infty
$$

(see [27, p. 294]). Clearly, if $\Gamma$ is $(p, J)$-non-trivial then $\Gamma$ is $(r, J)$-non-trivial for any $p \leq r \leq 1$.

Let $\Gamma$ be a $(p, J)$-non-trivial quasi-Banach sequence lattice and let $\bar{A}=$ $\left(A_{0}, A_{1}\right)$ be a $p$-normed quasi-Banach couple. The J-space $\bar{A}_{\Gamma ; J}=\left(A_{0}, A_{1}\right)_{\Gamma ; J}$ is formed by all sums $a=\sum_{m=-\infty}^{\infty} u_{m}$ (convergence in $\left.A_{0}+A_{1}\right)$, where $\left(u_{m}\right) \subseteq$ $A_{0} \cap A_{1}$ and $\left(J\left(2^{m}, u_{m}\right)\right) \in \Gamma$. The quasi-norm on $\bar{A}_{\Gamma ; J}$ is

$$
\|a\|_{\bar{A}_{\Gamma ; J}}=\|a\|_{\left(A_{0}, A_{1}\right)_{\Gamma ; J}}=\inf \left\{\left\|\left(J\left(2^{m}, u_{m}\right)\right)\right\|_{\Gamma}: a=\sum_{m=-\infty}^{\infty} u_{m}\right\}
$$


(see [27]). It holds

$$
A_{0} \cap A_{1} \hookrightarrow\left(A_{0}, A_{1}\right)_{\Gamma ; J} \hookrightarrow A_{0}+A_{1} .
$$

Moreover, $(\cdot, \cdot)_{\Gamma ; J}$ is an interpolation functor in the category of all $p$-normed quasi-Banach couples.

Remark 3.1. If $\Gamma$ is not $(p, J)$-non-trivial then the second embedding in (3.1) may fail. Indeed, consider the $p$-normed quasi-Banach couple $\left(\ell_{p}, \ell_{p}\left(2^{-m}\right)\right)$. If $\Gamma$ is not $(p, J)$-non-trivial then given any $N \in \mathbb{N}$, there is $x=\left(x_{m}\right) \in \Gamma$ and $L_{N} \in \mathbb{N}$ such that

$$
\|x\|_{\Gamma} \leq 1 \quad \text { and } \quad\left(\sum_{|m| \leq L_{N}}\left[\min \left(1,2^{-m}\right)\left|x_{m}\right|\right]^{p}\right)^{1 / p}>N .
$$

Put $y_{m}=x_{m}$ if $|m| \leq L_{N}$ and $y_{m}=0$ otherwise, and let $y=\left(y_{m}\right)$. Let $e_{k}=\left(\delta_{m}^{k}\right)$ where $\delta_{m}^{k}$ is the Kronecker's delta and write $u_{k}=y_{k} e_{k}, k \in \mathbb{Z}$. Then we have that $y=\sum_{m=-\infty}^{\infty} u_{m}\left(\right.$ convergence in $\left.\ell_{p}+\ell_{p}\left(2^{-m}\right)\right)$ with $J\left(2^{k}, u_{k} ; \ell_{p}, \ell_{p}\left(2^{-m}\right)\right)=\left|y_{k}\right|$. Hence

and

$$
\|y\|_{\left(\ell_{p}, \ell_{p}\left(2^{-m}\right)\right)_{\Gamma ; J}} \leq\|y\|_{\Gamma} \leq\|x\|_{\Gamma} \leq 1
$$

$$
\|y\|_{\ell_{p}+\ell_{p}\left(2^{-m}\right)} \sim\left(\sum_{|m| \leq L_{N}}\left[\min \left(1,2^{-m}\right)\left|x_{m}\right|\right]^{p}\right)^{1 / p}>N .
$$

This yields that $\left(\ell_{p}, \ell_{p}\left(2^{-m}\right)\right)_{\Gamma ; J}$ is not continuously embedded in $\ell_{p}+\ell_{p}\left(2^{-m}\right)$.

Note that a direct consequence of the definition of $\left(A_{0}, A_{1}\right)_{\Gamma ; J}$ is that

$$
\left(A_{0}, A_{1}\right)_{\Gamma ; J} \subseteq\left(A_{0}+A_{1}\right)^{\circ} .
$$

Next we take $\mathbb{A} \in \mathbb{R}^{2}, 0<q \leq \infty$ satisfying (2.1) and consider the $K$ method $(\cdot, \cdot)_{1, q, \mathbb{A}}$. We want to describe $(\cdot, \cdot)_{1, q, \mathbb{A}}$ as a $J$-functor.

Remark 3.2. It follows from Remark 2.1 and (3.2) that if (2.2) fails then $(\cdot, \cdot)_{1, q, \mathbb{A}}$ does not admit a representation as a $J$-method.

If $(2.1)$ and (2.2) holds, then for any $p$-normed quasi-Banach couple $\left(A_{0}, A_{1}\right)$ we have with equivalent quasi-norms

$$
\left(A_{0}, A_{1}\right)_{1, q, \mathbb{A}}=\left(A_{0}^{\sim}, A_{1}^{\sim}\right)_{\Lambda ; J} \text { where } \Lambda=\left(\ell_{p}, \ell_{p}\left(2^{-m}\right)\right)_{1, q, \mathbb{A}}
$$

(see [27, Theorem 3.19] and [6, Theorem 2.1]). Here $A_{j}^{\sim}$ stands for the Gagliardo completion of $A_{j}$, formed by all those $a \in A_{0}+A_{1}$ having a finite quasi-norm

$$
\|a\|_{A_{\tilde{j}}}=\sup \left\{t^{-j} K(t, a): t>0\right\}, j=0,1
$$

(see $[3,4])$.

The following result gives a more precise description of the quasi-norm of the sequence lattice $\Lambda$ of (3.3). 
Lemma 3.3. Let $0<p \leq 1,0<q \leq \infty$ and $\mathbb{A}=\left(\alpha_{0}, \alpha_{\infty}\right) \in \mathbb{R}^{2}$ satisfying (2.1) and (2.2). If $x=\left(x_{m}\right)_{m \in \mathbb{Z}}$ then

$$
\begin{gathered}
\|x\|_{\left(\ell_{p}, \ell_{p}\left(2^{-m}\right)\right)_{1, q, \mathbb{A}}} \sim\left(\sum_{m=-\infty}^{0}\left[\ell^{\alpha_{0}}\left(2^{m}\right)\left(\sum_{k=m}^{0} 2^{-k p}\left|x_{k}\right|^{p}\right)^{1 / p}\right]^{q}\right)^{1 / q} \\
+\left(\sum_{m=0}^{\infty}\left[\ell^{\alpha_{\infty}}\left(2^{m}\right)\left(\sum_{k=m}^{\infty} 2^{-k p}\left|x_{k}\right|^{p}\right)^{1 / p}\right]^{q}\right)^{1 / q} .
\end{gathered}
$$

Proof. Since $K_{p}\left(2^{k}, x ; \ell_{p}, \ell_{p}\left(2^{-m}\right)\right)=\left(\sum_{m=-\infty}^{\infty}\left[\min \left(1,2^{k-m}\right)\left|x_{m}\right|\right]^{p}\right)^{1 / p}$ we have

$$
\begin{aligned}
\|x\|_{\left(\ell_{p}, \ell_{p}\left(2^{-m}\right)\right)_{1, q, \mathbb{A}}} \sim\left(\sum_{m=-\infty}^{\infty}\left[2^{-m} \ell^{\mathbb{A}}\left(2^{m}\right)\left(\sum_{k=-\infty}^{\infty} \min \left(1,2^{m-k}\right)^{p}\left|x_{k}\right|^{p}\right)^{1 / p}\right]^{q}\right)^{1 / q} \\
\sim\left(\sum_{m=-\infty}^{0}\left[2^{-m} \ell^{\alpha_{0}}\left(2^{m}\right)\left(\sum_{k=-\infty}^{m}\left|x_{k}\right|^{p}\right)^{1 / p}\right]^{q}\right)^{1 / q} \\
+\left(\sum_{m=-\infty}^{0}\left[\ell^{\alpha_{0}}\left(2^{m}\right)\left(\sum_{k=m}^{0} 2^{-k p}\left|x_{k}\right|^{p}\right)^{1 / p}\right]^{q}\right)^{1 / q} \\
+\left(\sum_{m=-\infty}^{0}\left[\ell^{\alpha_{0}}\left(2^{m}\right)\left(\sum_{k=0}^{\infty} 2^{-k p}\left|x_{k}\right|^{p}\right)^{1 / p}\right]^{q}\right)^{1 / q} \\
+\left(\sum_{m=0}^{\infty}\left[2^{-m} \ell^{\alpha_{\infty}}\left(2^{m}\right)\left(\sum_{k=-\infty}^{0}\left|x_{k}\right|^{p}\right)^{1 / p}\right]^{q}\right)^{1 / q} \\
+\left(\sum_{m=0}^{\infty}\left[2^{-m} \ell^{\alpha_{\infty}}\left(2^{m}\right)\left(\sum_{k=0}^{m}\left|x_{k}\right|^{p}\right)^{1 / p}\right]^{q}\right)^{1 / q} \\
+\left(\sum_{m=0}^{\infty}\left[\ell^{\alpha_{\infty}}\left(2^{m}\right)\left(\sum_{k=m}^{\infty} 2^{-k p}\left|x_{k}\right|^{p}\right)^{1 / p}\right]^{q}\right)^{1 / q} \\
=S_{1}+S_{2}+S_{3}+S_{4}+S_{5}+S_{6} .
\end{aligned}
$$

Having in mind (2.1), we get $S_{3} \sim\left(\sum_{k=0}^{\infty} 2^{-k p}\left|x_{k}\right|^{p}\right)^{1 / p}$. Hence, the term in $S_{6}$ with $m=0$ shows that $S_{3} \lesssim S_{6}$. On the other hand,

$$
S_{4} \sim\left(\sum_{k=-\infty}^{0}\left|x_{k}\right|^{p}\right)^{1 / p} \leq S_{1}
$$

Next we check that $S_{1} \lesssim S_{2}$ and $S_{5} \lesssim S_{6}$. Assume first that $0<q \leq p$. Then

$$
S_{1} \leq\left(\sum_{m=-\infty}^{0} 2^{-m q} \ell^{\alpha_{0} q}\left(2^{m}\right) \sum_{k=-\infty}^{m}\left|x_{k}\right|^{q}\right)^{1 / q}=\left(\sum_{k=-\infty}^{0}\left|x_{k}\right|^{q} \sum_{m=k}^{0} 2^{-m q} \ell^{\alpha_{0} q}\left(2^{m}\right)\right)^{1 / q}
$$




$$
\lesssim\left(\sum_{k=-\infty}^{0}\left|x_{k}\right|^{q} 2^{-k q} \ell^{\alpha_{0} q}\left(2^{k}\right)\right)^{1 / q} \leq S_{2} .
$$

Similarly,

$$
\begin{aligned}
S_{5} & \leq\left(\sum_{m=0}^{\infty} 2^{-m q} \ell^{\alpha_{\infty} q}\left(2^{m}\right) \sum_{k=0}^{m}\left|x_{k}\right|^{q}\right)^{1 / q}=\left(\sum_{k=0}^{\infty}\left|x_{k}\right|^{q} \sum_{m=k}^{\infty} 2^{-m q} \ell_{\infty}^{\alpha_{\infty} q}\left(2^{m}\right)\right)^{1 / q} \\
& \lesssim\left(\sum_{k=0}^{\infty}\left|x_{k}\right|^{q} 2^{-k q} \ell^{\alpha_{\infty} q}\left(2^{k}\right)\right)^{1 / q} \leq S_{6} .
\end{aligned}
$$

Suppose now that $0<p<q<\infty$. Let $0<\varepsilon<1$. Using Hölder's inequality we get

$$
\begin{aligned}
\left(\sum_{k=-\infty}^{m}\left|x_{k}\right|^{p}\right)^{q / p} & \leq\left(\sum_{k=-\infty}^{m} 2^{-k(1-\varepsilon) q}\left|x_{k}\right|^{q}\right)\left(\sum_{k=-\infty}^{m} 2^{k(1-\varepsilon) p q /(q-p)}\right)^{(q-p) / p} \\
& \sim 2^{m(1-\varepsilon) q} \sum_{k=-\infty}^{m} 2^{-k(1-\varepsilon) q}\left|x_{k}\right|^{q}
\end{aligned}
$$

Hence,

$$
\begin{aligned}
S_{1} & \lesssim\left(\sum_{m=-\infty}^{0} 2^{-m \varepsilon q} \ell^{\alpha_{0} q}\left(2^{m}\right) \sum_{k=-\infty}^{m} 2^{-k(1-\varepsilon) q}\left|x_{k}\right|^{q}\right)^{1 / q} \\
& =\left(\sum_{k=-\infty}^{0} 2^{-k(1-\varepsilon) q}\left|x_{k}\right|^{q} \sum_{m=k}^{0} 2^{-m \varepsilon q} \ell^{\alpha_{0} q}\left(2^{m}\right)\right)^{1 / q} \\
& \lesssim\left(\sum_{k=-\infty}^{0} 2^{-k q}\left|x_{k}\right|^{q} \ell^{\alpha_{0} q}\left(2^{k}\right)\right)^{1 / q} \leq S_{2} .
\end{aligned}
$$

As for $S_{5}$, for the interior sum we obtain

$$
\begin{aligned}
\left(\sum_{k=0}^{m}\left|x_{k}\right|^{p}\right)^{q / p} & \leq\left(\sum_{k=0}^{m} 2^{-k q \varepsilon}\left|x_{k}\right|^{q}\right)\left(\sum_{k=0}^{m} 2^{k \varepsilon p q /(q-p)}\right)^{(q-p) / p} \\
& \sim 2^{m \varepsilon q} \sum_{k=0}^{m} 2^{-k q \varepsilon}\left|x_{k}\right|^{q}
\end{aligned}
$$

Therefore,

$$
S_{5} \lesssim\left(\sum_{m=0}^{\infty} 2^{-m q(1-\varepsilon)} \ell^{\alpha_{\infty} q}\left(2^{m}\right) \sum_{k=0}^{m} 2^{-k q \varepsilon}\left|x_{k}\right|^{q}\right)^{1 / q}
$$




$$
\begin{aligned}
& =\left(\sum_{k=0}^{\infty} 2^{-k q \varepsilon}\left|x_{k}\right|^{q} \sum_{m=k}^{\infty} 2^{-m q(1-\varepsilon)} \ell^{\alpha_{\infty} q}\left(2^{m}\right)\right)^{1 / q} \\
& \lesssim\left(\sum_{k=0}^{\infty} 2^{-k q}\left|x_{k}\right|^{q} \ell^{\alpha_{\infty} q}\left(2^{k}\right)\right)^{1 / q} \leq S_{6} .
\end{aligned}
$$

The case $q=\infty$ can be treated analogously. This completes the proof.

When $p=q$ the space $\Lambda$ is a weighted $\ell_{q}$-space as we show next.

Lemma 3.4. Let $0<q<\infty$ and $\mathbb{A}=\left(\alpha_{0}, \alpha_{\infty}\right) \in \mathbb{R}^{2}$ satisfying $\alpha_{0}+1 / q<0 \leq$ $\alpha_{\infty}+1 / q$. Then we have, with equivalence of quasi-norms

$$
\left(\ell_{q}, \ell_{q}\left(2^{-m}\right)\right)_{1, q, \mathbb{A}}= \begin{cases}\ell_{q}\left(2^{-m} \ell^{\mathbb{A}+1 / q}\left(2^{m}\right)\right) & \text { if } 0<\alpha_{\infty}+1 / q \\ \ell_{q}\left(2^{-m} \ell^{\mathbb{A}+1 / q}\left(2^{m}\right) \ell \ell^{(0,1 / q)}\left(2^{m}\right)\right) & \text { if } 0=\alpha_{\infty}+1 / q .\end{cases}
$$

Proof. According to Lemma 3.3, we obtain

$$
\begin{aligned}
\|x\|_{\left(\ell_{q}, \ell_{q}\left(2^{-m}\right)\right)_{1, q, \mathbb{A}}} \sim & \left(\sum_{m=-\infty}^{0} \ell^{\alpha_{0} q}\left(2^{m}\right) \sum_{k=m}^{0} 2^{-k q}\left|x_{k}\right|^{q}\right)^{1 / q} \\
& +\left(\sum_{m=0}^{\infty} \ell^{\alpha_{\infty} q}\left(2^{m}\right) \sum_{k=m}^{\infty} 2^{-k q}\left|x_{k}\right|^{q}\right)^{1 / q} .
\end{aligned}
$$

Changing the order of summation, we derive

$$
\begin{aligned}
\|x\|_{\left(\ell_{q}, \ell_{q}\left(2^{-m}\right)\right)_{1, q, \mathbb{A}}} \sim & \left(\sum_{k=-\infty}^{0} 2^{-k q}\left|x_{k}\right|^{q} \sum_{m=-\infty}^{k} \ell^{\alpha_{0} q}\left(2^{m}\right)\right)^{1 / q} \\
& +\left(\sum_{k=0}^{\infty} 2^{-k q}\left|x_{k}\right|^{q} \sum_{m=0}^{k} \ell^{\alpha_{\infty} q}\left(2^{m}\right)\right)^{1 / q} .
\end{aligned}
$$

Since

$$
\sum_{m=-\infty}^{k} \ell^{\alpha_{0} q}\left(2^{m}\right) \sim \ell^{\alpha_{0} q+1}\left(2^{k}\right) \text { if } \alpha_{0}+1 / q<0
$$

and

$$
\sum_{m=0}^{k} \ell_{\infty}^{\alpha_{\infty} q}\left(2^{m}\right) \sim \begin{cases}\ell^{\alpha_{\infty} q+1}\left(2^{k}\right) & \text { if } 0<\alpha_{\infty}+1 / q \\ \ell \ell\left(2^{k}\right) & \text { if } 0=\alpha_{\infty}+1 / q\end{cases}
$$

the result follows. 
A consequence of Lemma 3.4 is that the space $\ell_{q}\left(2^{-m} \ell^{\mathbb{A}+1 / q}\left(2^{m}\right)\right.$ ) (respectively, $\left.\ell_{q}\left(2^{-m} \ell^{\mathbb{A}+1 / q}\left(2^{m}\right) \ell \ell^{(0,1 / q)}\left(2^{m}\right)\right)\right)$ is continuously embedded in $\ell_{q}+\ell_{q}\left(2^{-m}\right)$, that is to say, it is a $(q, J)$-non-trivial lattice. Now we characterize this property for $p \neq q$ and $\mathbb{B} \neq(0,1 / q)$.

In what follows, given $0<p \leq 1$ and $0<q \leq \infty$, we put

$$
q^{*}= \begin{cases}\infty & \text { if } 0<q \leq p \\ \frac{p q}{q-p} & \text { if } 0<p<q<\infty \\ p & \text { if } q=\infty\end{cases}
$$

Observe that if $p=1$ and $1 \leq q \leq \infty$, then $q^{*}=q^{\prime}$ where $1 / q+1 / q^{\prime}=1$.

Lemma 3.5. Let $0<p \leq 1,0<q \leq \infty$ and $\mathbb{A}=\left(\alpha_{0}, \alpha_{\infty}\right), \mathbb{B}=\left(\beta_{0}, \beta_{\infty}\right) \in \mathbb{R}^{2}$. The necessary and sufficient condition for the continuous embedding

$$
\ell_{q}\left(2^{-m} \ell^{\mathbb{A}}\left(2^{m}\right) \ell \ell^{\mathbb{B}}\left(2^{m}\right)\right) \hookrightarrow \ell_{p}+\ell_{p}\left(2^{-m}\right)
$$

is that

$$
\begin{cases}\alpha_{\infty}>0, \text { or } \alpha_{\infty}=0 \text { and } \beta_{\infty} \geq 0 & \text { if } 0<q \leq p \\ \alpha_{\infty}+\frac{1}{q}-\frac{1}{p}>0, \text { or } \alpha_{\infty}+\frac{1}{q}-\frac{1}{p}=0 \text { and } \beta_{\infty}+\frac{1}{q}-\frac{1}{p}>0 & \text { if } p<q \leq \infty\end{cases}
$$

Proof. Take any $x=\left(x_{m}\right) \in \ell_{q}\left(2^{-m} \ell^{\mathbb{A}}\left(2^{m}\right) \ell \ell^{\mathbb{B}}\left(2^{m}\right)\right)$. Using Hölder's inequality we get

$$
\begin{aligned}
& \|x\|_{\ell_{p}+\ell_{p}\left(2^{-m}\right)} \sim\left(\sum_{m=-\infty}^{\infty} \min \left(1,2^{-m}\right)^{p}\left|x_{m}\right|^{p}\right)^{1 / p} \\
& \leq\left\|\left(\min \left(1,2^{-m}\right)\right)\right\|_{\ell_{q^{*}}\left(2^{m} \ell^{-\mathbb{A}}\left(2^{m}\right) \ell \ell^{-\mathbb{B}}\left(2^{m}\right)\right)}\|x\|_{\ell_{q}\left(2^{-m} \ell^{\mathbb{A}}\left(2^{m}\right) \ell \ell^{\mathbb{B}}\left(2^{m}\right)\right)} \\
& =\left(\sum_{m=-\infty}^{0}\left[2^{m} \ell^{-\alpha_{0}}\left(2^{m}\right) \ell \ell^{-\beta_{0}}\left(2^{m}\right)\right]^{q^{*}}+\sum_{m=0}^{\infty}\left[\ell^{-\alpha_{\infty}}\left(2^{m}\right) \ell \ell^{-\beta_{\infty}}\left(2^{m}\right)\right]^{q *}\right)^{1 / q^{*}} \\
& \quad \times\|x\|_{\ell_{q}\left(2^{-m} \ell^{\mathbb{A}}\left(2^{m}\right) \ell \ell^{\mathbb{B}}\left(2^{m}\right)\right)} .
\end{aligned}
$$

The last sums are finite by (3.4). Therefore we obtain that

$$
\ell_{q}\left(2^{-m} \ell^{\mathbb{A}}\left(2^{m}\right) \ell \ell^{\mathbb{B}}\left(2^{m}\right)\right) \hookrightarrow \ell_{p}+\ell_{p}\left(2^{-m}\right) .
$$

Conversely, if (3.4) does not hold then

$$
\begin{cases}\alpha_{\infty}<0, \text { or } \alpha_{\infty}=0 \text { and } \beta_{\infty}<0 & \text { if } 0<q \leq p \\ \alpha_{\infty}+\frac{1}{q}-\frac{1}{p}<0, \text { or } \alpha_{\infty}+\frac{1}{q}-\frac{1}{p}=0 \text { and } \beta_{\infty}+\frac{1}{q}-\frac{1}{p} \leq 0 & \text { if } p<q \leq \infty\end{cases}
$$




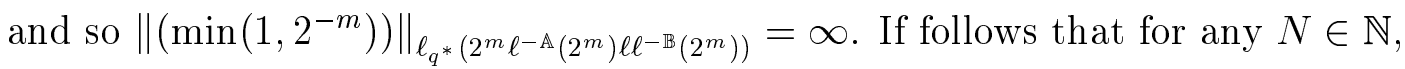
there is $L_{N} \in \mathbb{N}$ such that

$$
N<\left(\sum_{|m| \leq L_{N}}\left[\min \left(1,2^{-m}\right) 2^{m} \ell^{-\mathbb{A}}\left(2^{m}\right) \ell \ell^{-\mathbb{B}}\left(2^{m}\right)\right]^{q^{*}}\right)^{1 / q^{*}} .
$$

Assume now that $0<p<q<\infty$. Let

$$
y_{m}= \begin{cases}\min \left(1,2^{-m}\right)^{\frac{p}{q-p}} 2^{m \frac{q}{q-p}} \ell^{-\frac{q}{q-p} \mathbb{A}}\left(2^{m}\right) \ell \ell^{-\frac{q}{q-p} \mathbb{B}}\left(2^{m}\right) & \text { if }|m| \leq L_{N}, \\ 0 & \text { otherwise, }\end{cases}
$$

put $y=\left(y_{m}\right)$ and $x=\left(y_{m} /\|y\|_{\ell_{q}\left(2^{-m} \ell^{\mathbb{A}}\left(2^{m}\right) \ell \ell^{\mathbb{B}}\left(2^{m}\right)\right)}\right)$. Then $x$ belongs to the unit ball of $\ell_{q}\left(2^{-m} \ell^{\mathbb{A}}\left(2^{m}\right) \ell \ell^{\mathbb{B}}\left(2^{m}\right)\right)$ but

$$
\begin{aligned}
\|x\|_{\ell_{p}+\ell_{p}\left(2^{-m}\right)} & \sim\left(\sum_{m=-\infty}^{\infty}\left[\min \left(1,2^{-m}\right)\left|x_{m}\right|\right]^{p}\right)^{1 / p} \\
& =\frac{\left(\sum_{|m| \leq L_{N}}\left[\min \left(1,2^{-m}\right) 2^{m} \ell^{-\mathbb{A}}\left(2^{m}\right) \ell \ell^{-\mathbb{B}}\left(2^{m}\right)\right]^{q^{*}}\right)^{1 / p}}{\left(\sum_{|m| \leq L_{N}}\left[\min \left(1,2^{-m}\right) 2^{m} \ell^{-\mathbb{A}}\left(2^{m}\right) \ell \ell^{-\mathbb{B}}\left(2^{m}\right)\right]^{q^{*}}\right)^{1 / q}} \\
& =\left(\sum_{|m| \leq L_{N}}\left[\min \left(1,2^{-m}\right) 2^{m} \ell^{-\mathbb{A}}\left(2^{m}\right) \ell \ell^{-\mathbb{B}}\left(2^{m}\right)\right]^{q^{*}}\right)^{1 / q^{*}}>N .
\end{aligned}
$$

Hence, $\ell_{q}\left(2^{-m} \ell^{\mathbb{A}}\left(2^{m}\right) \ell \ell^{\mathbb{B}}\left(2^{m}\right)\right)$ is not continuously embedded in $\ell_{p}+\ell_{p}\left(2^{-m}\right)$.

In the case $q=\infty$, where $q^{*}=p$, we put

$$
y_{m}= \begin{cases}2^{m} \ell^{-\mathbb{A}}\left(2^{m}\right) \ell \ell^{-\mathbb{B}}\left(2^{m}\right) & \text { if }|m| \leq L_{N}, \\ 0 & \text { otherwise. }\end{cases}
$$

Then $\left\|\left(y_{m}\right)\right\|_{\ell_{\infty}\left(2^{-m} \ell^{\mathbb{A}}\left(2^{m}\right) \ell \ell^{\mathbb{B}}\left(2^{m}\right)\right)}=1$ but

$$
\left\|\left(y_{m}\right)\right\|_{\ell_{p}+\ell_{p}\left(2^{-m}\right)} \sim\left(\sum_{|m| \leq L_{N}}\left[\min \left(1,2^{-m}\right) 2^{m} \ell^{-\mathbb{A}}\left(2^{m}\right) \ell \ell^{-\mathbb{B}}\left(2^{m}\right)\right]^{p}\right)^{1 / p}>N .
$$

Finally, if $0<q<p$ we have $q^{*}=\infty$. We may assume that $L_{N}$ satisfies that

$$
\min \left(1,2^{-L_{N}}\right) 2^{L_{N}} \ell^{-\mathbb{A}}\left(2^{L_{N}}\right) \ell \ell^{-\mathbb{B}}\left(2^{L_{N}}\right)>N .
$$

Let $e_{L_{N}}=\left(\delta_{m}^{L_{N}}\right)$ and $x=2^{L_{N}} \ell^{-\mathbb{A}}\left(2^{L_{N}}\right) \ell \ell^{-\mathbb{B}}\left(2^{L_{N}}\right) e_{L_{N}}$. Then we have

$$
\begin{aligned}
& \|x\|_{\ell_{q}\left(2^{-m} \ell^{\mathbb{A}}\left(2^{m}\right) \ell \ell^{\mathbb{B}}\left(2^{m}\right)\right)}=1 \text { and } \\
& \|x\|_{\ell_{p}+\ell_{p}\left(2^{-m}\right)}=\min \left(1,2^{-L_{N}}\right) 2^{L_{N}} \ell^{-\mathbb{A}}\left(2^{L_{N}}\right) \ell \ell^{-\mathbb{B}}\left(2^{L_{N}}\right)>N .
\end{aligned}
$$

This completes the proof. 
Let $0<p \leq 1,0<q \leq \infty$ and $\mathbb{A}, \mathbb{B} \in \mathbb{R}^{2}$ satisfying (3.4). Given any $p$-normed quasi-Banach couple $\left(A_{0}, A_{1}\right)$, the logarithmic J-spaces are defined by

$$
\bar{A}_{1, q, \mathbb{A}}^{J}=\left(A_{0}, A_{1}\right)_{1, q, \mathbb{A}}^{J}=\left(A_{0}, A_{1}\right)_{\ell_{q}\left(2^{-m} \ell^{\mathbb{A}}\left(2^{m}\right)\right) ; J}
$$

and

$$
\bar{A}_{1, q, \mathbb{A}, \mathbb{B}}^{J}=\left(A_{0}, A_{1}\right)_{1, q, \mathbb{A}, \mathbb{B}}^{J}=\left(A_{0}, A_{1}\right)_{\ell_{q}\left(2^{-m} \ell^{\mathbb{A}}\left(2^{m}\right) \ell \ell^{\mathbb{B}}\left(2^{m}\right)\right) ; J} .
$$

We close this section by computing the characteristic function of the interpolation functor $(\cdot, \cdot)_{1, q, \mathbb{A}}^{J}$.

Lemma 3.6. Let $0<p \leq 1,0<q \leq \infty$ and $\mathbb{A}=\left(\alpha_{0}, \alpha_{\infty}\right) \in \mathbb{R}^{2}$ such that

$$
\begin{cases}\alpha_{\infty} \geq 0 & \text { if } 0<q \leq p \\ \alpha_{\infty}+\frac{1}{q}-\frac{1}{p}>0 & \text { if } 0<p<q \leq \infty\end{cases}
$$

For $k \in \mathbb{Z}$, put

$$
\mathrm{u}_{q, \mathbb{A}, p}\left(2^{k}\right)=\sup \left\{\left(\sum_{m=-\infty}^{\infty}\left[\min \left(1,2^{k-m}\right)\left|x_{m}\right|\right]^{p}\right)^{1 / p}:\left\|\left(x_{m}\right)\right\|_{\ell_{q}\left(2^{-m} \ell^{\mathbb{A}}\left(2^{m}\right)\right)} \leq 1\right\} .
$$

Then we have

$$
\mathrm{u}_{q, \mathbb{A}, p}\left(2^{k}\right)=\left\|\left(\min \left(1,2^{k-m}\right)\right)\right\|_{\ell_{q^{*}}\left(2^{m} \ell^{-\mathbb{A}}\left(2^{m}\right)\right)} .
$$

Proof. Let $x=\left(x_{m}\right) \in \ell_{q}\left(2^{-m} \ell^{\mathbb{A}}\left(2^{m}\right)\right)$ with $\|x\|_{\ell_{q}\left(2^{-m} \ell^{\mathbb{A}}\left(2^{m}\right)\right)} \leq 1$. Applying Hölder's inequality we get

$$
\left(\sum_{m=-\infty}^{\infty}\left[\min \left(1,2^{k-m}\right)\left|x_{m}\right|\right]^{p}\right)^{1 / p} \leq\left\|\left(\min \left(1,2^{k-m}\right)\right)\right\|_{\ell_{q^{*}\left(2^{m} \ell^{-\mathbb{A}}\left(2^{m}\right)\right)}}\|x\|_{\ell_{q}\left(2^{-m} \ell^{\mathbb{A}}\left(2^{m}\right)\right)}
$$

Therefore, $\mathrm{u}_{q, \mathbb{A}, p}\left(2^{k}\right) \leq\left\|\left(\min \left(1,2^{k-m}\right)\right)\right\|_{\ell_{q^{*}}\left(2^{m} \ell^{-\mathbb{A}}\left(2^{m}\right)\right)}$.

Conversely, if $0<q \leq p$, so $q^{*}=\infty$, given any $\varepsilon>0$ we can find $n \in \mathbb{Z}$ such that

$$
\min \left(1,2^{k-n}\right) 2^{n} \ell^{-\mathbb{A}}\left(2^{n}\right) \geq\left\|\left(\min \left(1,2^{k-m}\right)\right)\right\|_{\ell_{\infty}\left(2^{m} \ell^{-\mathbb{A}}\left(2^{m}\right)\right)}-\varepsilon .
$$

Take $x=2^{n} \ell^{-\mathbb{A}}\left(2^{n}\right) e_{n}$. Clearly $\|x\|_{\ell_{q}\left(2^{-m} \ell^{\mathbb{A}}\left(2^{m}\right)\right)}=1$. Moreover

$$
\begin{aligned}
\left(\sum_{m=-\infty}^{\infty}\left[\min \left(1,2^{k-m}\right)\left|x_{m}\right|\right]^{p}\right)^{1 / p} & =\min \left(1,2^{k-n}\right) 2^{n} \ell^{-\mathbb{A}}\left(2^{n}\right) \\
& >\left\|\left(\min \left(1,2^{k-m}\right)\right)\right\|_{\ell_{\infty}\left(2^{m} \ell^{-\mathbb{A}}\left(2^{m}\right)\right)}-\varepsilon
\end{aligned}
$$


Whence $\mathrm{u}_{q, \mathbb{A}, p}\left(2^{k}\right)=\left\|\left(\min \left(1,2^{k-m}\right)\right)\right\|_{\ell_{\infty}\left(2^{m} \ell^{-\mathbb{A}}\left(2^{m}\right)\right)}$.

Suppose now $0<p<q<\infty$. Let

$$
y_{m}=\min \left(1,2^{k-m}\right)^{\frac{p}{q-p}} 2^{m \frac{q}{q-p}} \ell^{-\frac{q}{q-p}} \mathbb{A}\left(2^{m}\right), m \in \mathbb{Z},
$$

and $y=\left(y_{m}\right)$. Then

$$
\|y\|_{\ell_{q}\left(2^{-m} \ell^{\mathbb{A}}\left(2^{m}\right)\right)}=\left\|\left(\min \left(1,2^{k-m}\right)\right)\right\|_{\ell_{q^{*}}\left(2^{m} \ell^{-\mathbb{A}}\left(2^{m}\right)\right)}^{p /(q-p)} .
$$

Put

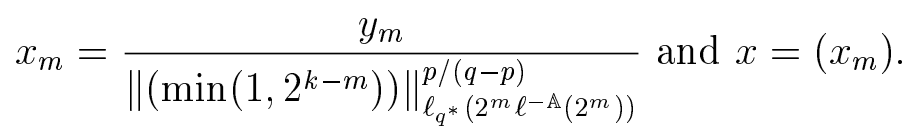

Then, $\|x\|_{\ell_{q}\left(2^{-m} \ell^{\mathbb{A}}\left(2^{m}\right)\right)}=1$ and

$$
\begin{aligned}
\left(\sum_{m=-\infty}^{\infty}\left[\min \left(1,2^{k-m}\right)\left|x_{m}\right|\right]^{p}\right)^{1 / p} & =\frac{\left(\sum_{m=-\infty}^{\infty}\left[\min \left(1,2^{k-m}\right) 2^{m} \ell^{-\mathbb{A}}\left(2^{m}\right)\right]^{q^{*}}\right)^{1 / p}}{\left(\sum_{m=-\infty}^{\infty}\left[\min \left(1,2^{k-m}\right) 2^{m} \ell^{-\mathbb{A}}\left(2^{m}\right)\right]^{q^{*}}\right)^{1 / q}} \\
& =\left\|\left(\min \left(1,2^{k-m}\right)\right)\right\|_{\ell_{q^{*}}\left(2^{m} \ell^{-\mathbb{A}}\left(2^{m}\right)\right)} .
\end{aligned}
$$

Consequently,

$$
\mathrm{u}_{q, \mathbb{A}, p}\left(2^{k}\right)=\left\|\left(\min \left(1,2^{k-m}\right)\right)\right\|_{\ell_{q^{*}}\left(2^{m} \ell^{-\mathbb{A}}\left(2^{m}\right)\right)} .
$$

Finally, assume that $q=\infty$ so $q^{*}=p$. Let $x_{m}=2^{m} \ell^{-\mathbb{A}}\left(2^{m}\right), m \in \mathbb{Z}$. We have $\left\|\left(x_{m}\right)\right\|_{\ell_{\infty}\left(2^{-m} \ell^{\mathbb{A}}\left(2^{m}\right)\right)}=1$ and

$$
\left(\sum_{m=-\infty}^{\infty}\left[\min \left(1,2^{k-m}\right)\left|x_{m}\right|\right]^{p}\right)^{1 / p}=\left(\sum_{m=-\infty}^{\infty}\left[\min \left(1,2^{k-m}\right) 2^{m} \ell^{-\mathbb{A}}\left(2^{m}\right)\right]^{p}\right)^{1 / p}
$$

This yields that

$$
\mathrm{u}_{q, \mathbb{A}, p}\left(2^{k}\right)=\left\|\left(\min \left(1,2^{k-m}\right)\right)\right\|_{\ell_{p}\left(2^{m} \ell^{-\mathbb{A}}\left(2^{m}\right)\right)} .
$$

Proposition 3.7. Let $A$ be a p-normed quasi-Banach space, let $0<q \leq \infty$ and $\mathbb{A}=\left(\alpha_{0}, \alpha_{\infty}\right) \in \mathbb{R}^{2}$ satisfying (3.5). For any $k \in \mathbb{Z}$, we have

$$
\left(A, 2^{-k} A\right)_{1, q, \mathbb{A}}^{J} \hookrightarrow \mathrm{u}_{q, \mathbb{A}, p}\left(2^{k}\right)^{-1} A,
$$

being the norm of the embedding less than or equal to 1 . Moreover, if in addition we suppose that $p=1$ or $0<q \leq p$, then we have with equal quasi-norms

$$
\left(A, 2^{-k} A\right)_{1, q, \mathbb{A}}^{J}=\mathrm{u}_{q, \mathbb{A}, p}\left(2^{k}\right)^{-1} A .
$$


Proof. Observe that $J\left(2^{m}, u ; A, 2^{-k} A\right)=\max \left(1,2^{m-k}\right)\|u\|_{A}$. If $\left(u_{m}\right) \subseteq A$ and $a=\sum_{m=-\infty}^{\infty} u_{m}$ (convergence in $A$ ), we obtain

$$
\begin{aligned}
\|a\|_{A} \leq & \left(\sum_{m=-\infty}^{\infty}\left\|u_{m}\right\|_{A}^{p}\right)^{1 / p}=\left(\sum_{m=-\infty}^{\infty}\left[\min \left(1,2^{k-m}\right) J\left(2^{m}, u_{m}\right)\right]^{p}\right)^{1 / p} \\
\leq & \left(\sum _ { m = - \infty } ^ { \infty } \left[\min \left(1,2^{k-m}\right) \frac{J\left(2^{m}, u_{m}\right)}{\left.\left.\left\|\left(J\left(2^{m}, u_{m}\right)\right)\right\|_{\ell_{q}\left(2^{-m} \ell_{\left.\ell^{\mathbb{A}}\left(2^{m}\right)\right)}\right.}\right]^{p}\right)^{1 / p}}\right.\right. \\
& \times\left\|\left(J\left(2^{m}, u_{m}\right)\right)\right\|_{\ell_{q}\left(2^{-m} \ell^{\mathbb{A}}\left(2^{m}\right)\right)} \\
\leq & \mathrm{u}_{q, \mathbb{A}, p}\left(2^{k}\right)\left\|\left(J\left(2^{m}, u_{m}\right)\right)\right\|_{\ell_{q}\left(2^{-m} \ell^{\mathbb{A}}\left(2^{m}\right)\right)} .
\end{aligned}
$$

This yields that the embedding $\left(A, 2^{-k} A\right)_{1, q, \mathbb{A}}^{J} \hookrightarrow \mathrm{u}_{q, \mathbb{A}, p}\left(2^{k}\right)^{-1} A$ has a norm less than or equal to 1 .

Conversely, if $p=1$, given any $\varepsilon>0$ we can find $x=\left(x_{m}\right)$ such that $\|x\|_{\ell_{q}\left(2^{-m} \ell^{\mathbb{A}}\left(2^{m}\right)\right)} \leq 1$ and

$$
\mathrm{u}_{q, \mathbb{A}, 1}\left(2^{k}\right)-\varepsilon<\sum_{m=-\infty}^{\infty} \min \left(1,2^{k-m}\right)\left|x_{m}\right|
$$

We can represent any $a \in A$ as $a=\sum_{m=-\infty}^{\infty} u_{m}$ with

$$
u_{m}=\frac{\min \left(1,2^{k-m}\right)\left|x_{m}\right|}{\sum_{m=-\infty}^{\infty} \min \left(1,2^{k-m}\right)\left|x_{m}\right|} a, m \in \mathbb{Z} .
$$

Then

$$
J\left(2^{m}, u_{m}\right)=\frac{\left|x_{m}\right|}{\sum_{m=-\infty}^{\infty} \min \left(1,2^{k-m}\right)\left|x_{m}\right|}\|a\|_{A} \leq \frac{\left|x_{m}\right|}{\mathrm{u}_{q, \mathbb{A}, 1}\left(2^{k}\right)-\varepsilon}\|a\|_{A} .
$$

Whence

$$
\|a\|_{\left(A, 2^{-k} A\right)_{1, q, \mathbb{A}}^{J}} \leq\left(\mathrm{u}_{q, \mathbb{A}, 1}\left(2^{k}\right)-\varepsilon\right)^{-1}\|a\|_{A} .
$$

This yields that the embedding $\mathrm{u}_{q, \mathbb{A}, 1}\left(2^{k}\right)^{-1} A \hookrightarrow\left(A, 2^{-k} A\right)_{1, q, \mathbb{A}}^{J}$ has norm less than or equal to 1.

Suppose now that $0<q \leq p$. Then $q^{*}=\infty$. Given any $\varepsilon>0$ we can find $n \in \mathbb{Z}$ such that

$$
\mathrm{u}_{q, \mathbb{A}, p}\left(2^{k}\right)-\varepsilon<2^{n} \ell^{-\mathbb{A}}\left(2^{n}\right) \min \left(1,2^{k-n}\right) .
$$

For any $a \in A$ we obtain

$$
J\left(2^{n}, a\right)=\frac{1}{\min \left(1,2^{k-n}\right)}\|a\|_{A}<\left(\mathrm{u}_{q, \mathbb{A}, p}\left(2^{k}\right)-\varepsilon\right)^{-1} 2^{n} \ell^{-\mathbb{A}}\left(2^{n}\right)\|a\|_{A} .
$$


Consider the representation $a=\sum_{m=-\infty}^{\infty} \delta_{m}^{n} a$. We have

$$
\|a\|_{\left(A, 2^{-k} A\right)_{1, q, \mathbb{A}}^{J}} \leq 2^{-n} \ell^{\mathbb{A}}\left(2^{n}\right) J\left(2^{n}, a\right)<\left(\mathrm{u}_{q, \mathbb{A}, p}\left(2^{k}\right)-\varepsilon\right)^{-1}\|a\|_{A} .
$$

This completes the proof.

Corollary 3.8. Let $A$ be a p-normed quasi-Banach space, let $k \in \mathbb{Z}, 0<q \leq \infty$ and $\mathbb{A}=\left(\alpha_{0}, \alpha_{\infty}\right) \in \mathbb{R}^{2}$ satisfying (3.5). The following equalities hold with equivalence of quasi-norms:

For $p=1$,

$$
\left(A, 2^{-k} A\right)_{1, q, \mathbb{A}}^{J}= \begin{cases}2^{-k} \ell^{\mathbb{A}}\left(2^{k}\right) A & \text { if } 0<q \leq 1 \text { and } \alpha_{0} \leq 0, \\ 2^{-k} \ell^{\left(0, \alpha_{\infty}\right)}\left(2^{k}\right) A & \text { if } 0<q \leq 1 \text { and } \alpha_{0}>0, \\ 2^{-k} \ell^{\mathbb{A}-1 / q^{*}}\left(2^{k}\right) A & \text { if } 1<q \leq \infty \text { and } \alpha_{0}<\frac{1}{q^{*}}, \\ 2^{-k} \ell^{\mathbb{A}-1 / q^{*}}\left(2^{k}\right) \ell \ell^{\left(-1 / q^{*}, 0\right)}\left(2^{k}\right) A & \text { if } 1<q \leq \infty \text { and } \alpha_{0}=\frac{1}{q^{*}}, \\ 2^{-k} \ell^{\left(0, \alpha_{\infty}-1 / q^{*}\right)}\left(2^{k}\right) A & \text { if } 1<q \leq \infty \text { and } \alpha_{0}>\frac{1}{q^{*}} .\end{cases}
$$

For $0<q \leq p$,

$$
\left(A, 2^{-k} A\right)_{1, q, \mathbb{A}}^{J}= \begin{cases}2^{-k} \ell^{\mathbb{A}}\left(2^{k}\right) A & \text { if } \alpha_{0} \leq 0 \\ 2^{-k} \ell^{\left(0, \alpha_{\infty}\right)}\left(2^{k}\right) A & \text { if } \alpha_{0}>0 .\end{cases}
$$

The constants in the equivalence depend on $p$ but they are independent of $k \in \mathbb{Z}$ and of the concrete p-normed space A.

Proof. Let $\tilde{\mathbb{A}}=\left(\alpha_{\infty}, \alpha_{0}\right)$. Using Lemma 3.6 and a change of variables we can relate $\mathrm{u}_{q, \mathbb{A}, p}$ with the function $\mathrm{v}_{q^{*},-\tilde{\mathbb{A}}}$ defined in Lemma 2.2. We have

$$
\begin{aligned}
\mathrm{u}_{q, \mathbb{A}, p}\left(2^{k}\right) & =\left\|\left(\min \left(1,2^{k-m}\right)\right)\right\|_{\ell_{q^{*}}\left(2^{m} \ell^{-\mathbb{A}}\left(2^{m}\right)\right)} \\
& =\left\|\left(\min \left(1,2^{k+m}\right)\right)\right\|_{\ell_{q^{*}}\left(2^{-m} \ell^{-\tilde{\mathbb{A}}}\left(2^{m}\right)\right)}=\mathrm{v}_{q^{*},-\tilde{\mathbb{A}}}\left(2^{-k}\right) .
\end{aligned}
$$

Now the result follows by applying Proposition 3.7 and Lemma 2.2.

\section{The equivalence theorems}

In this section we investigate the description of $\left(A_{0}, A_{1}\right)_{1, q, \mathbb{A}}$ in terms of the $J$-functional and the logarithmic sequence spaces $\ell_{q}\left(2^{-m} \ell^{\mathbb{M}}\left(2^{m}\right)\right)$. Fix $0<p \leq$ 1. We assume that the functors $(\cdot, \cdot)_{1, q, \mathbb{A}}$ and $(\cdot, \cdot)_{1, q, \mathbb{M}}^{J}$ are well-defined in the category of $p$-normed quasi-Banach couples. This means that $\mathbb{A}=\left(\alpha_{0}, \alpha_{\infty}\right)$ and $q$ should satisfy (2.1), and $\mathbb{M}=\left(\mu_{0}, \mu_{\infty}\right), q$ and $p$ should satisfy (3.4). In addition, according to (3.2) and Remark 2.1, $\mathbb{A}$ and $q$ should also satisfy (2.2). So, the problem to study reads as follows: 
Given $0<p \leq 1, \mathbb{A}=\left(\alpha_{0}, \alpha_{\infty}\right) \in \mathbb{R}^{2}$ and $0<q \leq \infty$ such that

$$
\begin{cases}\alpha_{0}+1 / q<0 \leq \alpha_{\infty}+1 / q & \text { if } 0<q<\infty, \\ \alpha_{0} \leq 0<\alpha_{\infty} & \text { if } q=\infty,\end{cases}
$$

find $\mathbb{M}=\left(\mu_{0}, \mu_{\infty}\right) \in \mathbb{R}^{2}$ with

$$
\begin{cases}\mu_{\infty} \geq 0 & \text { if } 0<q \leq p \\ \mu_{\infty}>\frac{1}{p}-\frac{1}{q} & \text { if } 0<p<q \leq \infty\end{cases}
$$

and such that for any $p$-normed quasi-Banach couple $\bar{A}=\left(A_{0}, A_{1}\right)$ we have

$$
\left(A_{0}, A_{1}\right)_{1, q, \mathbb{A}}=\left(A_{0}, A_{1}\right)_{1, q, \mathbb{M}}^{J}
$$

with equivalence of quasi-norms where the constants are independent of $\bar{A}$.

Unfortunately, for some values of parameters $p, q$ and $\mathbb{A}$ there is no $\mathbb{M}$ satisfying (4.3). Take, for example, $0<p<q \leq 1$ and $\alpha_{0}+1 / q<0<$ $\alpha_{\infty}+1 / q \leq 1 / p-1 / q$. Let $\mathbb{M}=\left(\mu_{0}, \mu_{\infty}\right)$ with $\mu_{\infty}>1 / p-1 / q$. If (4.3) holds then Proposition 2.3 and Corollary 3.8 with $A=\ell_{1}$ would yield

$$
\mathrm{u}_{q, \mathbb{M}, 1}\left(2^{k}\right)^{-1} \sim \mathrm{v}_{q, \mathbb{A}}\left(2^{k}\right)
$$

with constant in the equivalence independent of $k$. By Lemma 2.2 and Corollary 3.8 we get for $k \in \mathbb{Z}, k$ positive, that $\mathrm{v}_{q, \mathbb{A}}\left(2^{k}\right) \sim 2^{-k} \ell^{\alpha_{\infty}+1 / q}\left(2^{k}\right)$ and $\mathrm{u}_{q, \mathbb{M}, 1}\left(2^{k}\right)^{-1} \sim 2^{-k} \ell^{\mu_{\infty}}\left(2^{k}\right)$. It follows that $\alpha_{\infty}+1 / q=\mu_{\infty}$. Hence $\alpha_{\infty}+1 / q>$ $1 / p-1 / q$ which contradicts that $\alpha_{\infty}+1 / q \leq 1 / p-1 / q$.

This example leads us to investigate the weaker questions of finding the best $\mathbb{M}$ and $\mathbb{B}$ such that

$$
\left(A_{0}, A_{1}\right)_{1, q, \mathbb{A}} \hookrightarrow\left(A_{0}, A_{1}\right)_{1, q, \mathbb{M}}^{J}
$$

or

$$
\left(A_{0}, A_{1}\right)_{1, q, \mathbb{B}}^{J} \hookrightarrow\left(A_{0}, A_{1}\right)_{1, q, \mathbb{A}}
$$

for any $p$-normed quasi-Banach couple $\left(A_{0}, A_{1}\right)$ and with constants in the embeddings independent of $\left(A_{0}, A_{1}\right)$.

In the case $0<p<q \leq 1, \alpha_{0}+1 / q<0$ and $1 / p-1 / q<\alpha_{\infty}+1 / q$, if we assume that (4.4) holds for some $\mathbb{M}=\left(\mu_{0}, \mu_{\infty}\right)$ with $\mu_{\infty}>1 / p-1 / q$, then proceeding as above we obtain that $\mathrm{v}_{q, \mathbb{A}}\left(2^{k}\right) \ell_{1} \hookrightarrow \mathrm{u}_{q, \mathbb{M}, 1}\left(2^{k}\right)^{-1} \ell_{1}$. The values of $\mathrm{v}_{q, \mathbb{A}}\left(2^{k}\right)$ and $\mathrm{u}_{q, \mathbb{M}, 1}\left(2^{k}\right)^{-1}$ have been pointed out above. Since the embedding is valid for any positive $k$, we get $\mu_{\infty} \leq \alpha_{\infty}+1 / q$. Let now $k \in \mathbb{Z}, k$ negative, then $\mathrm{v}_{q, \mathbb{A}}\left(2^{k}\right) \sim 2^{-k} \ell^{\alpha_{0}+1 / q}\left(2^{k}\right)$ while

$$
\mathrm{u}_{q, \mathbb{M}, 1}\left(2^{k}\right)^{-1} \sim \begin{cases}2^{-k} \ell^{\mu_{0}}\left(2^{k}\right) & \text { if } \mu_{0} \leq 0 \\ 2^{-k} & \text { if } \mu_{0}>0\end{cases}
$$


The option $2^{-k}$ is not possible since $\alpha_{0}+1 / q<0$. Hence, we should have $\mu_{0} \leq \alpha_{0}+1 / q$. In other words, for these $p, q$ and $\mathbb{A}$, the best possible $\mathbb{M}$ would be $\left(\alpha_{0}+1 / q, \alpha_{\infty}+1 / q\right)$. Next we show that (4.4) holds for this choice of $\mathbb{M}$. We shall use that $\left(A_{0}, A_{1}\right)_{1, q, \mathbb{A}}=\left(A_{0}^{\sim}, A_{1}^{\sim}\right)_{1, q, \mathbb{A}}$. This equality follows from the equivalence

$$
K\left(t, a ; A_{0}^{\sim}, A_{1}^{\sim}\right) \leq K\left(t, a ; A_{0}, A_{1}\right) \leq \max \left\{c_{A_{0}}, c_{A_{1}}\right\} K\left(t, a ; A_{0}^{\sim}, A_{1}^{\sim}\right)
$$

which can be established by doing minor modifications in the arguments of [3, Theorem V.I.5].

Theorem 4.1. Let $0<p<q \leq 1$ and $\mathbb{A}=\left(\alpha_{0}, \alpha_{\infty}\right) \in \mathbb{R}^{2}$ satisfying

$$
\alpha_{0}+1 / q<0, \alpha_{\infty}+1 / q>1 / p-1 / q
$$

Then, for any p-normed quasi-Banach couple $\bar{A}=\left(A_{0}, A_{1}\right)$, we have

$$
\left(A_{0}, A_{1}\right)_{1, q, \mathbb{A}} \hookrightarrow\left(A_{0}^{\sim}, A_{1}^{\sim}\right)_{1, q, \mathbb{A}+1 / q}^{J} .
$$

Proof. According to (3.3), we get

$$
\left(A_{0}, A_{1}\right)_{1, q, \mathbb{A}}=\left(A_{0}^{\sim}, A_{1}^{\sim}\right)_{1, q, \mathbb{A}}=\left(A_{0}^{\sim}, A_{1}^{\sim}\right)_{\left(\ell_{p}, \ell_{p}\left(2^{-m}\right)\right)_{1, q, \mathbb{A}} ; J} .
$$

Besides, since $p<q$, we have $\ell_{p} \hookrightarrow \ell_{q}$ and $\ell_{p}\left(2^{-m}\right) \hookrightarrow \ell_{q}\left(2^{-m}\right)$. Therefore,

$$
\left(\ell_{p}, \ell_{p}\left(2^{-m}\right)\right)_{1, q, \mathbb{A}} \hookrightarrow\left(\ell_{q}, \ell_{q}\left(2^{-m}\right)\right)_{1, q, \mathbb{A}}=\ell_{q}\left(2^{-m} \ell^{\mathbb{A}+1 / q}\left(2^{m}\right)\right)
$$

where the last equality follows from Lemma 3.4. Consequently,

$$
\left(A_{0}, A_{1}\right)_{1, q, \mathbb{A}} \hookrightarrow\left(A_{0}^{\sim}, A_{1}^{\sim}\right)_{1, q, \mathbb{A}+1 / q}^{J} .
$$

Next we consider the case $1 \leq q \leq \infty$. This time our arguments are based on decompositions of the type considered in [12] and [16].

Theorem 4.2. Let $0<p \leq 1 \leq q \leq \infty$ and $\mathbb{A}=\left(\alpha_{0}, \alpha_{\infty}\right) \in \mathbb{R}^{2}$ satisfying

$$
\alpha_{0}+1 / q<0 \text { and } \alpha_{\infty}+1 / q>1 / p-1 \text {. }
$$

Then, for any $p$-Banach couple $\bar{A}=\left(A_{0}, A_{1}\right)$, we have

$$
\left(A_{0}, A_{1}\right)_{1, q, \mathbb{A}} \hookrightarrow\left(A_{0}, A_{1}\right)_{1, q, \mathbb{A}+1}^{J} .
$$


Proof. Take any $a \in\left(A_{0}, A_{1}\right)_{1, q, \mathbb{A}}$. It follows from (4.7) (see $\left.(2.2)\right)$ that $a \in$ $\left(A_{0}+A_{1}\right)^{\circ}$. Hence,

$$
\min \left(1, t^{-1}\right) K(t, a) \rightarrow 0 \text { as } t \rightarrow 0 \text { and as } t \rightarrow \infty .
$$

For $\nu \in \mathbb{Z}$, let

$$
\gamma_{\nu}= \begin{cases}2^{-2^{-\nu-1}} & \text { if } \nu<0 \\ 1 & \text { if } \nu=0 \\ 2^{2^{\nu-1}} & \text { if } \nu>0\end{cases}
$$

Choose $a_{0, \nu} \in A_{0}, a_{1, \nu} \in A_{1}$ such that $a=a_{0, \nu}+a_{1, \nu}$ and

$$
\left\|a_{0, \nu}\right\|_{A_{0}}^{p}+\gamma_{\nu-1}^{p}\left\|a_{1, \nu}\right\|_{A_{1}}^{p} \leq 2^{p} K_{p}\left(\gamma_{\nu-1}, a\right)^{p} .
$$

Let $u_{\nu}=a_{0, \nu}-a_{0, \nu-1}=a_{1, \nu-1}-a_{1, \nu} \in A_{0} \cap A_{1}$. We have

$$
\begin{aligned}
\left\|a-\sum_{\nu=N}^{M} u_{\nu}\right\|_{A_{0}+A_{1}}^{p} & =\left\|a+a_{0, N-1}-a_{0, M}\right\|_{A_{0}+A_{1}}^{p} \\
& =\left\|a_{1, M}+a_{0, N-1}\right\|_{A_{0}+A_{1}}^{p} \\
& \leq 2^{p}\left(\gamma_{M-1}^{-p} K_{p}\left(\gamma_{M-1}, a\right)^{p}+K_{p}\left(\gamma_{N-2}, a\right)^{p}\right) \rightarrow 0
\end{aligned}
$$

as $M \rightarrow \infty$ and $N \rightarrow-\infty$. So $a=\sum_{\nu=-\infty}^{\infty} u_{\nu}$. Moreover, $\left\|u_{\nu}\right\|_{A_{0}+A_{1}} \rightarrow 0$ as $\nu \rightarrow-\infty$ and as $\nu \rightarrow \infty$. Besides,

$$
\frac{J\left(\gamma_{\nu-1}, u_{\nu}\right)}{\gamma_{\nu-1}} \lesssim \frac{K_{p}\left(\gamma_{\nu-2}, a\right)}{\gamma_{\nu-2}}, \nu \in \mathbb{Z}
$$

Indeed,

$$
\begin{aligned}
\frac{J\left(\gamma_{\nu-1}, u_{\nu}\right)^{p}}{\gamma_{\nu-1}^{p}} & \leq \gamma_{\nu-1}^{-p}\left(\left\|u_{\nu}\right\|_{A_{0}}^{p}+\gamma_{\nu-1}^{p}\left\|u_{\nu}\right\|_{A_{1}}^{p}\right) \\
& \leq \gamma_{\nu-1}^{-p}\left(\left\|a_{0, \nu}\right\|_{A_{0}}^{p}+\left\|a_{0, \nu-1}\right\|_{A_{0}}^{p}+\gamma_{\nu-1}^{p}\left\|a_{1, \nu}\right\|_{A_{1}}^{p}+\gamma_{\nu-1}^{p}\left\|a_{1, \nu-1}\right\|_{A_{1}}^{p}\right) \\
& \leq 2^{p} \gamma_{\nu-1}^{-p}\left(K_{p}\left(\gamma_{\nu-1}, a\right)^{p}+\frac{\gamma_{\nu-1}^{p}}{\gamma_{\nu-2}^{p}} K_{p}\left(\gamma_{\nu-2}, a\right)^{p}\right) \lesssim \frac{K_{p}\left(\gamma_{\nu-2}, a\right)^{p}}{\gamma_{\nu-2}^{p}}
\end{aligned}
$$

where we have used in the last inequality that the function $t^{-1} K(t, a)$ is nonincreasing.

For $\nu \in \mathbb{Z}$, put $I_{\nu}=\left[\gamma_{\nu-1}, \gamma_{\nu}\right)$. If $2^{m} \in I_{\nu}$, let $w_{m}$ be $u_{\nu}$ divided by the number of $m$ such that $2^{m} \in I_{\nu}$. That is

$$
w_{m}= \begin{cases}\frac{u_{\nu}}{2^{-\nu-1}} & \text { if } 2^{m} \in I_{\nu} \text { and } \nu<0 \\ u_{\nu} & \text { if } 2^{m} \in I_{\nu} \text { and } \nu=0,1 \\ \frac{u_{\nu}}{2^{\nu-2}} & \text { if } 2^{m} \in I_{\nu} \text { and } \nu>1\end{cases}
$$


This sequence also satisfies that $\left(w_{m}\right) \subseteq A_{0} \cap A_{1}$ with $a=\sum_{m=-\infty}^{\infty} w_{m}$ because for some $0 \leq d, f<1$ we have

$$
\begin{aligned}
\left\|a-\sum_{m=N}^{M} w_{m}\right\|_{A_{0}+A_{1}}^{p} & =\left\|a-\sum_{\nu=P}^{Q} u_{\nu}-d u_{P-1}-f u_{Q+1}\right\|_{A_{0}+A_{1}}^{p} \\
& \leq\left\|a-\sum_{\nu=P}^{Q} u_{\nu}\right\|_{A_{0}+A_{1}}^{p}+\left\|u_{P-1}\right\|_{A_{0}+A_{1}}^{p}+\left\|u_{Q+1}\right\|_{A_{0}+A_{1}}^{p} \rightarrow 0
\end{aligned}
$$

as $N \rightarrow-\infty$ and $M \rightarrow \infty$. Consequently, using (4.8), we derive if $1 \leq q<\infty$

$$
\begin{aligned}
\|a\|_{\left(A_{0}, A_{1}\right)_{1, q, \mathbb{A}+1}^{J}} & \leq\left(\sum_{m=-\infty}^{\infty} 2^{-m q} \ell^{\mathbb{A} q+q}\left(2^{m}\right) J\left(2^{m}, w_{m}\right)^{q}\right)^{1 / q} \\
& \sim\left(\sum_{\nu=-\infty}^{\infty} \sum_{2^{m} \in I_{\nu}} 2^{-|\nu| q} \frac{J\left(2^{m}, u_{\nu}\right)^{q}}{2^{m q}} \ell^{\mathbb{A} q+q}\left(2^{m}\right)\right)^{1 / q} \\
& \lesssim\left(\sum_{\nu=-\infty}^{\infty} \sum_{2^{m} \in I_{\nu}} 2^{-|\nu| q} \frac{J\left(\gamma_{\nu-1}, u_{\nu}\right)^{q}}{\gamma_{\nu-1}^{q}} 2^{|\nu|(\mathbb{A} q+q)}\right)^{1 / q} \\
& \lesssim\left(\sum_{\nu=-\infty}^{\infty} \sum_{2^{m} \in I_{\nu}} \frac{K_{p}\left(\gamma_{\nu-2}, a\right)^{q}}{\gamma_{\nu-2}^{q}} 2^{|\nu| \mathbb{A} q}\right)^{1 / q} \\
& \sim\left(\sum_{\nu=-\infty}^{\infty} \sum_{2^{m} \in I_{\nu-2}} \frac{K_{p}\left(\gamma_{\nu-2}, a\right)^{q}}{\gamma_{\nu-2}^{q}} 2^{|\nu| \mathbb{A} q}\right)^{1 / q} \\
& \lesssim\left(\sum_{\nu=-\infty}^{\infty} \sum_{2^{m} \in I_{\nu-2}} \frac{K_{p}\left(2^{m}, a\right)^{q}}{2^{m q}} \ell^{\mathbb{A} q}\left(2^{m}\right)\right)^{1 / q} \\
& =\|a\|_{\left(A_{0}, A_{1}\right)_{1, q, \mathbb{A}}} \cdot
\end{aligned}
$$

The case $q=\infty$ can be treated similarly.

Next we show that the embedding in Theorem 4.2 is the best possible. We rely on the characteristic functions of the functors.

Let $0<p<1<q<\infty$ and $\mathbb{A}, \mathbb{M} \in \mathbb{R}^{2}$ satisfying (4.1) and (4.2). If (4.4) holds then Proposition 2.3 and Corollary 3.8 with $A=\ell_{1}$ yield that

$$
\mathrm{u}_{q, \mathbb{M}, 1}\left(2^{k}\right)^{-1} \lesssim \mathrm{v}_{q, \mathbb{A}}\left(2^{k}\right) .
$$

Take any $k \in \mathbb{Z}, k$ negative. Using Lemma 2.2 we have that $\mathrm{v}_{q, \mathbb{A}}\left(2^{k}\right)=$ $2^{-k} \ell^{\alpha_{0}+1 / q}\left(2^{k}\right)$ and by Corollary 3.8 we get

$$
\mathrm{u}_{q, \mathbb{M}, 1}\left(2^{k}\right)^{-1} \sim \begin{cases}2^{-k} \ell^{\mu_{0}-1+1 / q}\left(2^{k}\right) & \text { if } \mu_{0}<1-1 / q \\ 2^{-k} \ell \ell^{-1+1 / q}\left(2^{k}\right) & \text { if } \mu_{0}=1-1 / q \\ 2^{-k} & \text { if } \mu_{0}>1-1 / q .\end{cases}
$$


Hence, (4.9) is only possible if $\mu_{0} \leq \alpha_{0}+1$. Consider now $k$ positive. Then

$$
\mathrm{v}_{q, \mathbb{A}}\left(2^{k}\right) \sim \begin{cases}2^{-k} \ell^{\alpha_{\infty}+1 / q}\left(2^{k}\right) & \text { if } \alpha_{\infty}+1 / q>0 \\ 2^{-k} \ell \ell^{1 / q}\left(2^{k}\right) & \text { if } \alpha_{\infty}+1 / q=0\end{cases}
$$

and $\mathrm{u}_{q, \mathbb{M}, 1}\left(2^{k}\right)^{-1} \sim 2^{-k} \ell^{\mu_{\infty}-1+1 / q}\left(2^{k}\right)$. Since $\mu_{\infty}>1 / p-1 / q>1-1 / q$, we derive that if (4.9) holds, then $\mu_{\infty} \leq \alpha_{\infty}+1$ and that there is no $\mathbb{M}$ if $0 \leq \alpha_{\infty}+1 / q \leq$ $1 / p-1$. Consequently, the embedding in Theorem 4.2 is optima. Note that these arguments also explain the assumption on $\alpha_{\infty}$ in the statement of the theorem. The cases $p=1<q \leq \infty, 0<p<1$ with $q=\infty$ and $0<p<1=q$ can be treated analogously.

Now we turn our attention to embeddings of the type (4.5).

Theorem 4.3. Let $0<p \leq 1,0<p<q \leq \infty$ and $\mathbb{A}=\left(\alpha_{0}, \alpha_{\infty}\right) \in \mathbb{R}^{2}$ satisfying

$$
\alpha_{0}+1 / q<0<\alpha_{\infty}+1 / q .
$$

Then, for any $p$-Banach couple $\bar{A}=\left(A_{0}, A_{1}\right)$, we have

$$
\left(A_{0}, A_{1}\right)_{1, q, \mathbb{A}+1 / p}^{J} \hookrightarrow\left(A_{0}, A_{1}\right)_{1, q, \mathbb{A}} .
$$

Proof. Take any $a \in\left(A_{0}, A_{1}\right)_{1, q, \mathbb{A}+1 / p}^{J}$ and let $\left(u_{m}\right) \subseteq A_{0} \cap A_{1}$ such that $a=$ $\sum_{m=-\infty}^{\infty} u_{m}$ and $\left\|\left(J\left(2^{m}, u_{m}\right)\right)\right\|_{\ell_{q}\left(2^{-m} \ell^{\mathbb{A}+1 / p}\left(2^{m}\right)\right)} \leq 2\|a\|_{\left(A_{0}, A_{1}\right)_{1, q, \mathbb{A}+1 / p}^{J}}$. Then

$$
\begin{aligned}
\|a\|_{\left(A_{0}, A_{1}\right)_{1, q, \mathbb{A}}} & \leq\left(\sum_{k=-\infty}^{\infty}\left[2^{-k} \ell^{\mathbb{A}}\left(2^{k}\right)\left(\sum_{m=-\infty}^{\infty} \min \left(1,2^{k-m}\right)^{p} J\left(2^{m}, u_{m}\right)^{p}\right)^{1 / p}\right]^{q}\right)^{1 / q} \\
\sim & \left\|\left(J\left(2^{m}, u_{m}\right)\right)\right\|_{\left(\ell_{p}, \ell_{p}\left(2^{-m}\right)\right)_{1, q, \mathbb{A}}} \\
\sim & \left(\sum_{k=-\infty}^{0}\left[\ell^{\alpha_{0}}\left(2^{k}\right)\left(\sum_{m=k}^{0} 2^{-m p} J\left(2^{m}, u_{m}\right)^{p}\right)^{1 / p}\right]^{q}\right)^{1 / q} \\
& \quad+\left(\sum_{k=0}^{\infty}\left[\ell^{\alpha_{\infty}}\left(2^{k}\right)\left(\sum_{m=k}^{\infty} 2^{-m p} J\left(2^{m}, u_{m}\right)^{p}\right)^{1 / p}\right]^{q}\right)^{1 / q}
\end{aligned}
$$

where we have used Lemma 3.3 in the last equivalence. Suppose now that $q<\infty$. To estimate the first term take any $0<\varepsilon<-\left(\alpha_{0}+1 / q\right)$. Using Hölder's inequality, for the interior sum of the first term, we get

$$
\begin{gathered}
\left(\sum_{m=k}^{0} 2^{-m p} J\left(2^{m}, u_{m}\right)^{p}\right)^{q / p} \leq\left(\sum_{m=k}^{0} 2^{-m q} J\left(2^{m}, u_{m}\right)^{q} \ell^{\alpha_{0} q+q / p+\varepsilon q}\left(2^{m}\right)\right) \\
\times\left(\sum_{m=k}^{0} \ell^{-q p\left(\alpha_{0}+1 / p+\varepsilon\right) /(q-p)}\left(2^{m}\right)\right)^{(q-p) / p}
\end{gathered}
$$




$$
\lesssim \ell^{-\alpha_{0} q-\varepsilon q-1}\left(2^{k}\right)\left(\sum_{m=k}^{0} 2^{-m q} J\left(2^{m}, u_{m}\right)^{q} \ell^{\alpha_{0} q+q / p+\varepsilon q}\left(2^{m}\right)\right)
$$

where we have used the condition on $\varepsilon$ in the last estimate. Whence, changing the order of summation, we derive

$$
\begin{aligned}
\left(\sum_{k=-\infty}^{0}[\right. & \left.\left.\ell^{\alpha_{0}}\left(2^{k}\right)\left(\sum_{m=k}^{0} 2^{-m p} J\left(2^{m}, u_{m}\right)^{p}\right)^{1 / p}\right]^{q}\right)^{1 / q} \\
& \leq\left(\sum_{k=-\infty}^{0} \ell^{-\varepsilon q-1}\left(2^{k}\right)\left(\sum_{m=k}^{0} 2^{-m q} J\left(2^{m}, u_{m}\right)^{q} \ell^{\alpha_{0} q+q / p+\varepsilon q}\left(2^{m}\right)\right)\right)^{1 / q} \\
& =\left(\sum_{m=-\infty}^{0} 2^{-m q} J\left(2^{m}, u_{m}\right)^{q} \ell^{\alpha_{0} q+q / p+\varepsilon q}\left(2^{m}\right) \sum_{k=-\infty}^{m} \ell^{-\varepsilon q-1}\left(2^{k}\right)\right)^{1 / q} \\
& \sim\left(\sum_{m=-\infty}^{0} 2^{-m q} J\left(2^{m}, u_{m}\right)^{q} \ell^{\alpha_{0} q+q / p}\left(2^{m}\right)\right)^{1 / q} \\
& \lesssim\|a\|_{\left(A_{0}, A_{1}\right)_{1, q, \mathrm{~A}+1 / p}^{J}} .
\end{aligned}
$$

For the second term, we choose now any $0<\varepsilon<\alpha_{\infty}+1 / q$ and we proceed similarly. We get

$$
\begin{gathered}
\left(\sum_{m=k}^{\infty} 2^{-m p} J\left(2^{m}, u_{m}\right)^{p}\right)^{q / p} \leq\left(\sum_{m=k}^{\infty} 2^{-m q} J\left(2^{m}, u_{m}\right)^{q} \ell^{\alpha_{\infty} q+q / p-\varepsilon q}\left(2^{m}\right)\right) \\
\times\left(\sum_{m=k}^{\infty} \ell^{-p q\left(\alpha_{\infty}+1 / p-\varepsilon\right) /(q-p)}\left(2^{m}\right)\right){ }^{(q-p) / p} \\
\sim \ell^{-\alpha_{\infty} q-1+\varepsilon q}\left(2^{k}\right)\left(\sum_{m=k}^{\infty} 2^{-m q} J\left(2^{m}, u_{m}\right)^{q} \ell^{\alpha_{\infty} q+q / p-\varepsilon q}\left(2^{m}\right)\right) .
\end{gathered}
$$

Therefore

$$
\begin{aligned}
\left(\sum _ { k = 0 } ^ { \infty } \left[\ell^{\alpha_{\infty}}\right.\right. & \left.\left.\left(2^{k}\right)\left(\sum_{m=k}^{\infty} 2^{-m p} J\left(2^{m}, u_{m}\right)^{p}\right)^{1 / p}\right]^{q}\right)^{1 / q} \\
& \lesssim\left(\sum_{k=0}^{\infty} \ell^{-1+\varepsilon q}\left(2^{k}\right)\left(\sum_{m=k}^{\infty} 2^{-m q} J\left(2^{m}, u_{m}\right)^{q} \ell^{\alpha_{\infty} q+q / p-\varepsilon q}\left(2^{m}\right)\right)\right)^{1 / q} \\
& =\left(\sum_{m=0}^{\infty} 2^{-m q} J\left(2^{m}, u_{m}\right)^{q} \ell^{\alpha_{\infty} q+q / p-\varepsilon q}\left(2^{m}\right)\left(\sum_{k=0}^{m} \ell^{-1+\varepsilon q}\left(2^{k}\right)\right)\right)^{1 / q} \\
& \lesssim\left(\sum_{m=0}^{\infty} 2^{-m q} J\left(2^{m}, u_{m}\right)^{q} \ell^{\alpha_{\infty} q+q / p}\left(2^{m}\right)\right)^{1 / q}
\end{aligned}
$$




$$
\lesssim\|a\|_{\left(A_{0}, A_{1}\right)_{1, q, \mathbb{A}+1 / p}^{J}}
$$

Consequently,

$$
\left(A_{0}, A_{1}\right)_{1, q, \mathbb{A}+1 / p}^{J} \hookrightarrow\left(A_{0}, A_{1}\right)_{1, q, \mathbb{A}} .
$$

The case $q=\infty$ can be treated analogously.

Next we show that the embedding of Theorem 4.3 is the best possible. For this aim we need some preparation.

Lemma 4.4. Let $0<p \leq 1,0<q \leq \infty$ and take any $\mathbb{B}=\left(\beta_{0}, \beta_{\infty}\right) \in \mathbb{R}^{2}$ with

$$
\begin{cases}\beta_{\infty} \geq 0 & \text { if } 0<q \leq p \\ \beta_{\infty}>\frac{1}{p}-\frac{1}{q} & \text { if } 0<p<q \leq \infty .\end{cases}
$$

Then

$$
\ell_{q}\left(2^{-m} \ell^{\mathbb{B}}\left(2^{m}\right)\right) \hookrightarrow\left(\ell_{p}, \ell_{p}\left(2^{-m}\right)\right)_{1, q, \mathbb{B}}^{J}
$$

Proof. Take any $x=\left(x_{m}\right) \in \ell_{q}\left(2^{-m} \ell^{\mathbb{B}}\left(2^{m}\right)\right)$ and $e_{k}=\left(\delta_{m}^{k}\right), k \in \mathbb{Z}$. Then

$$
J\left(2^{k}, x_{k} e_{k} ; \ell_{p}, \ell_{p}\left(2^{-m}\right)\right)=\left|x_{k}\right| \text { and } x=\sum_{m=-\infty}^{\infty} x_{m} e_{m} .
$$

Whence

$$
\begin{aligned}
\|x\|_{\left(\ell_{p}, \ell_{p}\left(2^{-m}\right)\right)_{1, q, \mathbb{B}}^{J}} & \leq\left\|\left(J\left(2^{k}, x_{k} e_{k} ; \ell_{p}, \ell_{p}\left(2^{-m}\right)\right)\right)\right\|_{\ell_{q}\left(2^{-m} \ell^{\mathbb{B}}\left(2^{m}\right)\right)} \\
& =\|x\|_{\ell_{q}\left(2^{-m} \ell^{\mathbb{B}}\left(2^{m}\right)\right)} .
\end{aligned}
$$

Proposition 4.5. Let $0<p \leq 1,0<q \leq \infty$ and $\mathbb{A}=\left(\alpha_{0}, \alpha_{\infty}\right), \mathbb{B}=\left(\beta_{0}, \beta_{\infty}\right) \in$ $\mathbb{R}^{2}$ satisfying (2.1) and (4.11). Then the embedding

$$
\ell_{q}\left(2^{-m} \ell^{\mathbb{B}}\left(2^{m}\right)\right) \hookrightarrow\left(\ell_{p}, \ell_{p}\left(2^{-m}\right)\right)_{1, q, \mathbb{A}}
$$

is a necessary and sufficient condition for $\left(A_{0}, A_{1}\right)_{1, q, \mathbb{B}}^{J} \hookrightarrow\left(A_{0}, A_{1}\right)_{1, q, \mathbb{A}}$ for any p-Banach couple $\left(A_{0}, A_{1}\right)$.

Proof. The condition is necessary by Lemma 4.4. Let us check that the condition is sufficient. Take any $p$-Banach couple $\left(A_{0}, A_{1}\right)$. For any $a \in\left(A_{0}, A_{1}\right)_{1, q, \mathbb{B}}^{J}$ we can find a representation $a=\sum_{m=-\infty}^{\infty} u_{m}$ with $\left(u_{m}\right) \subseteq A_{0} \cap A_{1}$ satisfying

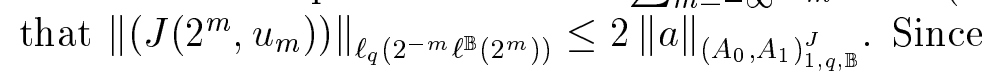

$$
K\left(2^{k}, a\right) \leq K_{p}\left(2^{k}, a\right) \leq\left(\sum_{m=-\infty}^{\infty} K_{p}\left(2^{k}, u_{m}\right)^{p}\right)^{1 / p}
$$




$$
\leq\left(\sum_{m=-\infty}^{\infty} \min \left(1,2^{k-m}\right)^{p} J\left(2^{m}, u_{m}\right)^{p}\right)^{1 / p}
$$

we obtain

$$
\begin{aligned}
\|a\|_{\left(A_{0}, A_{1}\right)_{1, q, \mathbb{A}}} & \leq\left\|\left(K\left(2^{k}, a\right)\right)\right\|_{\ell_{q}\left(2^{-k} \ell^{\mathbb{A}}\left(2^{k}\right)\right)} \\
& \leq\left\|\left(\sum_{m=-\infty}^{\infty} \min \left(1,2^{k-m}\right)^{p} J\left(2^{m}, u_{m}\right)^{p}\right)^{1 / p}\right\|_{\ell_{q}\left(2^{-k} \ell^{\mathbb{A}}\left(2^{k}\right)\right)} \\
& \sim \|\left(K\left(2^{k},\left(J\left(2^{m}, u_{m}\right)\right) ; \ell_{p}, \ell_{p}\left(2^{-m}\right)\right) \|_{\ell_{q}\left(2^{-k} \ell^{\mathbb{A}}\left(2^{k}\right)\right)}\right. \\
& =\left\|\left(J\left(2^{m}, u_{m}\right)\right)\right\|_{\left(\ell_{p}, \ell_{p}\left(2^{-m}\right)\right)_{1, q, \mathbb{A}}} .
\end{aligned}
$$

Now using the condition we derive that

$$
\begin{aligned}
\|a\|_{\left(A_{0}, A_{1}\right)_{1, q, \mathbb{A}}} & \lesssim\left\|\left(J\left(2^{m}, u_{m}\right)\right)\right\|_{\ell_{q}\left(2^{-m} \ell^{\mathbb{B}}\left(2^{m}\right)\right)} \\
& \leq 2\|a\|_{\left(A_{0}, A_{1}\right)_{1, q, \mathbb{B}}^{J}} .
\end{aligned}
$$

The next result refers to the operators

$$
\begin{aligned}
& H_{1} x=\left(\sum_{n=1}^{k}\left(1+\log 2^{k}\right)^{\alpha_{0} p}\left(1+\log 2^{n}\right)^{-\beta_{0} p} x_{n}\right)_{k \in \mathbb{N}}, \\
& H_{2} x=\left(\sum_{n=k}^{\infty}\left(1+\log 2^{k}\right)^{\alpha_{\infty} p}\left(1+\log 2^{n}\right)^{-\beta_{\infty} p} x_{n}\right)_{k \in \mathbb{N}}
\end{aligned}
$$

Here $x=\left(x_{n}\right)_{n \in \mathbb{N}}$. We consider $H_{1}$ and $H_{2}$ acting on the space $\boldsymbol{\ell}_{\mathbf{r}}$ of $r$-summable sequences with $\mathbb{N}$ as index set.

Lemma 4.6. Let $0<p \leq 1,0<q \leq \infty$ and $\mathbb{A}=\left(\alpha_{0}, \alpha_{\infty}\right), \mathbb{B}=\left(\beta_{0}, \beta_{\infty}\right) \in \mathbb{R}^{2}$ satisfying (2.1) and (4.11). Assume also that

$$
\ell_{q}\left(2^{-m} \ell^{\mathbb{B}}\left(2^{m}\right)\right) \hookrightarrow\left(\ell_{p}, \ell_{p}\left(2^{-m}\right)\right)_{1, q, \mathbb{A}} .
$$

Then $H_{1}$ and $H_{2}$ are bounded on $\boldsymbol{\ell}_{\mathbf{q} / \mathbf{p}}$.

Proof. Take any $x=\left(x_{n}\right) \in \boldsymbol{\ell}_{\mathbf{q} / \mathbf{p}}$. For $k \in \mathbb{Z}$, let

$$
y_{k}= \begin{cases}\left|x_{-k}\right|^{1 / p} 2^{k}\left(1-\log 2^{k}\right)^{-\beta_{0}} & \text { if } k \leq-1, \\ 0 & \text { if } k \geq 0,\end{cases}
$$

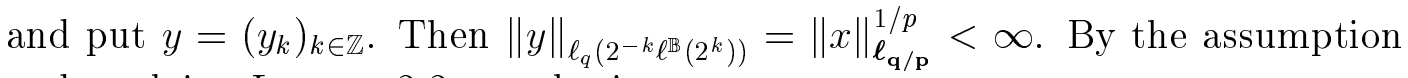
and applying Lemma 3.3, we obtain

$$
\|x\|_{\ell_{\mathbf{q} / \mathbf{p}}}^{1 / p}=\|y\|_{\ell_{q}\left(2^{-k} \ell^{\mathbb{B}}\left(2^{k}\right)\right)} \gtrsim\|y\|_{\left(\ell_{p}, \ell_{p}\left(2^{-m}\right)\right)_{1, q, \mathbb{A}}}
$$




$$
\begin{aligned}
\sim & \left(\sum_{k=-\infty}^{0}\left[\ell^{\alpha_{0}}\left(2^{k}\right)\left(\sum_{m=k}^{0} 2^{-m p}\left|y_{m}\right|^{p}\right)^{1 / p}\right]^{q}\right)^{1 / q} \\
& \quad+\left(\sum_{k=0}^{\infty}\left[\ell^{\alpha_{\infty}}\left(2^{k}\right)\left(\sum_{m=k}^{\infty} 2^{-m p}\left|y_{m}\right|^{p}\right)^{1 / p}\right]^{q}\right)^{1 / q} \\
= & \left(\sum_{k=-\infty}^{-1}\left[\left(1-\log 2^{k}\right)^{\alpha_{0}}\left(\sum_{m=k}^{-1}\left(1-\log 2^{m}\right)^{-\beta_{0} p}\left|x_{-m}\right|\right)^{1 / p}\right]^{q}\right)^{1 / q} \\
= & \left(\sum_{k=1}^{\infty}\left[\left(1+\log 2^{k}\right)^{\alpha_{0}}\left(\sum_{m=1}^{k}\left(1+\log 2^{m}\right)^{-\beta_{0} p}\left|x_{m}\right|\right)^{1 / p}\right]^{q}\right)^{1 / q} \\
\geq & \left\|H_{1} x\right\|_{\ell_{\mathbf{q} / \mathbf{p}}}^{1 / p} .
\end{aligned}
$$

Now we return our attention to $H_{2}$. Let $z=\left(z_{k}\right)_{k \in \mathbb{Z}}$ where

$$
z_{k}= \begin{cases}0 & \text { if } k \leq 0 \\ \left|x_{k}\right|^{1 / p} 2^{k}\left(1+\log 2^{k}\right)^{-\beta_{\infty}} & \text { if } k \geq 1\end{cases}
$$

Using again the assumption and Lemma 3.3, we derive

$$
\begin{aligned}
\|x\|_{\ell_{\mathbf{q} / \mathbf{p}}}^{1 / p} & =\|z\|_{\ell_{q}\left(2^{-k} \ell^{\mathbb{B}}\left(2^{k}\right)\right)} \gtrsim\|z\|_{\left(\ell_{p}, \ell_{p}\left(2^{-m}\right)\right)_{1, q, \mathbb{A}}} \\
& \gtrsim\left(\sum_{k=1}^{\infty}\left[\left(1+\log 2^{k}\right)^{\alpha_{\infty}}\left(\sum_{m=k}^{\infty} 2^{-m p}\left|z_{m}\right|^{p}\right)^{1 / p}\right]^{q}\right)^{1 / q} \\
& =\left(\sum_{k=1}^{\infty}\left[\left(1+\log 2^{k}\right)^{\alpha_{\infty}}\left(\sum_{m=k}^{\infty}\left(1+\log 2^{m}\right)^{-\beta_{\infty} p}\left|x_{m}\right|\right)^{1 / p}\right]^{q}\right)^{1 / q} \\
& \geq\left\|H_{2} x\right\|_{\ell_{\mathbf{q} / \mathbf{p}}}^{1 / p} .
\end{aligned}
$$

We shall also need some results on matrix transformations of $\boldsymbol{\ell}_{\mathbf{r}}$-spaces established by G. Bennett $[1,2]$. Let $\left(a_{n}\right),\left(b_{n}\right)$ be sequences of non-negative numbers and let $\mathbf{M}=\left(a_{n k}\right)$ with

$$
a_{n k}= \begin{cases}a_{n} b_{k} & \text { if } 1 \leq k \leq n \\ 0 & \text { if } k>n\end{cases}
$$

Put $T x=\left(\sum_{k=1}^{\infty} a_{n k} x_{k}\right)_{n \in \mathbb{N}}=\left(a_{n} \sum_{k=1}^{\infty} b_{k} x_{k}\right)_{n \in \mathbb{N}}$ for the operator defined by M. Let $1 \leq r \leq \infty$ and $1 / r+1 / r^{\prime}=1$. According to [1, Theorem 2] and 
[2, Theorem 1], the operator $T: \boldsymbol{\ell}_{\mathbf{r}} \rightarrow \boldsymbol{\ell}_{\mathbf{r}}$ is bounded if and only if

$$
\begin{cases}\sup _{N \in \mathbb{N}}\left(\sum_{n=N}^{\infty} a_{n}^{r}\right)^{1 / r}\left(\sum_{k=1}^{N} b_{k}^{r^{\prime}}\right)^{1 / r^{\prime}}<\infty & \text { for } 1<r<\infty \\ \sup _{N \in \mathbb{N}}\left(\sum_{n=N}^{\infty} a_{n}^{r}\right)^{1 / r} b_{N}<\infty & \text { for } r=1, \\ \sup _{N \in \mathbb{N}} a_{N} \sum_{k=1}^{N} b_{k}<\infty & \text { for } r=\infty\end{cases}
$$

Put $\widehat{\mathbf{M}}=\left(\widehat{a}_{n k}\right)$ with

$$
\widehat{a}_{n k}= \begin{cases}0 & \text { if } k<n \\ a_{k} b_{n} & \text { if } k \geq n\end{cases}
$$

and let $\widehat{T} x=\left(\sum_{k=1}^{\infty} \widehat{a}_{n k} x_{k}\right)_{n \in \mathbb{N}}=\left(b_{n} \sum_{k=n}^{\infty} a_{k} x_{k}\right)_{n \in \mathbb{N}}$. Since $\widehat{T}$ is the adjoint of the operator $T$, it follows from (4.12) that $\widehat{T}: \ell_{\mathbf{r}} \rightarrow \ell_{\mathbf{r}}$ is bounded if and only if

$$
\sup _{N \in \mathbb{N}}\left(\sum_{n=N}^{\infty} a_{n}^{r^{\prime}}\right)^{1 / r^{\prime}}\left(\sum_{k=1}^{N} b_{k}^{r}\right)^{1 / r}<\infty \text { for } 1<r<\infty .
$$

Furthermore, a direct computation shows that $\widehat{T}: \boldsymbol{\ell}_{\infty} \rightarrow \boldsymbol{\ell}_{\infty}$ is bounded if and only if

$$
\sup _{N \in \mathbb{N}} b_{N} \sum_{k=N}^{\infty} a_{k}<\infty .
$$

Observe that $H_{1}$ is the transformation defined by the matrix $\mathbf{M}=\left(a_{n k}\right)$ given by the sequences

$$
a_{n}=\left(1+\log 2^{n}\right)^{\alpha_{0} p} \text { and } b_{k}=\left(1+\log 2^{k}\right)^{-\beta_{0} p}
$$

while $H_{2}$ is the transformation defined by $\widehat{\mathbf{M}}$ with

$$
a_{k}=\left(1+\log 2^{k}\right)^{-\beta_{\infty} p} \text { and } b_{n}=\left(1+\log 2^{n}\right)^{\alpha_{\infty} p} .
$$

Now we are ready to show that the embedding of Theorem 4.3 is the best possible.

Proposition 4.7. Let $0<p \leq 1,0<p<q \leq \infty$, $\mathbb{A}=\left(\alpha_{0}, \alpha_{\infty}\right)$, $\mathbb{B}=$ $\left(\beta_{0}, \beta_{\infty}\right) \in \mathbb{R}^{2}$ satisfying (4.10) and (4.11) and consider the $p$-Banach couple $\left(\ell_{p}, \ell_{p}\left(2^{-m}\right)\right)$. If $\left(\ell_{p}, \ell_{p}\left(2^{-m}\right)\right)_{1, q, \mathbb{B}}^{J} \hookrightarrow\left(\ell_{p}, \ell_{p}\left(2^{-m}\right)\right)_{1, q, \mathbb{A}}$ then $\beta_{0} \geq \alpha_{0}+1 / p$ and $\beta_{\infty} \geq \alpha_{\infty}+1 / p$.

Proof. By the assumptions on parameters and Lemma 4.4 we have that

$$
\ell_{q}\left(2^{-m} \ell^{\mathbb{B}}\left(2^{m}\right)\right) \hookrightarrow\left(\ell_{p}, \ell_{p}\left(2^{-m}\right)\right)_{1, q, \mathbb{A}} .
$$


Hence, according to Lemma 4.6, operators $H_{1}$ and $H_{2}$ are bounded on $\boldsymbol{\ell}_{\mathbf{q} / \mathbf{p}}$. If $q<\infty$, applying (4.12) to $H_{1}$ we obtain that

$$
\sup _{N \in \mathbb{N}}\left(\sum_{n=N}^{\infty}\left(1+\log 2^{n}\right)^{\alpha_{0} q}\right)^{p / q}\left(\sum_{k=1}^{N}\left(1+\log 2^{k}\right)^{-\beta_{0} \frac{p q}{q-p}}\right)^{\frac{q-p}{p}}<\infty .
$$

Using that $\alpha_{0}+1 / q<0$, it follows that

$$
\left(\sum_{k=1}^{N}\left(1+\log 2^{k}\right)^{-\beta_{0} \frac{p q}{q-p}}\right)^{(q-p) / p} \lesssim\left(1+\log 2^{n}\right)^{-\alpha_{0}-1 / q} .
$$

Whence

$$
\left\{\begin{array}{ll}
\left(1+\log 2^{N}\right)^{-\beta_{0}+1 / p-1 / q} & \text { if } \beta_{0}<1 / p-1 / q \\
\left(1+\log \left(1+\log 2^{N}\right)\right) & \text { if } \beta_{0}=1 / p-1 / q \\
1 & \text { if } \beta_{0}>1 / p-1 / q .
\end{array}\right\} \lesssim\left(1+\log 2^{n}\right)^{-\alpha_{0}-1 / q}
$$

Since $-\alpha_{0}-1 / q>0$, it follows that

$$
\beta_{0} \geq 1 / p-1 / q, \text { or } \beta_{0}<1 / p-1 / q \text { and }-\beta_{0}+1 / p-1 / q \leq-\alpha_{0}-1 / q .
$$

Consequently, in both cases $\beta_{0} \geq \alpha_{0}+1 / p$.

On the other hand, since $H_{2}$ is also bounded on $\boldsymbol{\ell}_{\mathbf{q} / \mathbf{p}}$, according to (4.13), we obtain

$$
\sup _{N \in \mathbb{N}}\left(\sum_{n=N}^{\infty}\left(1+\log 2^{n}\right)^{-\beta_{\infty} \frac{p q}{q-p}}\right)^{(q-p) / q}\left(\sum_{k=1}^{N}\left(1+\log 2^{k}\right)^{\alpha_{\infty} q}\right)^{p / q}<\infty .
$$

Having in mind that $\beta_{\infty}>1 / p-1 / q$ and $\alpha_{\infty}+1 / q>0$, we get

$$
\left(1+\log 2^{N}\right)^{\alpha_{\infty}+1 / q} \lesssim\left(1+\log 2^{N}\right)^{\beta_{\infty}-1 / p+1 / q} .
$$

This yields that $\beta_{\infty} \geq \alpha_{\infty}+1 / p$.

The proof for $q=\infty$ is similar but using now (4.14).

The next result complements Theorem 4.3. It corresponds to the limit case $\alpha_{\infty}+1 / q=0$. One can establish it by using similar arguments to those of Theorem 4.3.

Theorem 4.8. Let $0<p \leq 1,0<p<q<\infty$ and $\mathbb{A}=\left(\alpha_{0}, \alpha_{\infty}\right) \in \mathbb{R}^{2}$ satisfying

$$
\alpha_{0}+1 / q<0=\alpha_{\infty}+1 / q .
$$

Then, for any $p$-Banach couple $\bar{A}=\left(A_{0}, A_{1}\right)$, we have

$$
\left(A_{0}, A_{1}\right)_{1, q, \mathbb{A}+1 / p,(0,1 / p)}^{J} \hookrightarrow\left(A_{0}, A_{1}\right)_{1, q, \mathbb{A}} .
$$


Using the previous ideas based on matrix transformations of $\boldsymbol{\ell}_{\mathbf{r}}$-spaces, one can also show that the embedding in Theorem 4.8 is the best possible.

Writing down Theorems 4.2 and 4.3 for $1 \leq q \leq \infty$ and $\bar{A}$ a Banach couple, so $p=1$, we obtain the following result of Cobos and Segurado [16, Theorem $3.5]$.

Corollary 4.9. Let $\mathbb{A}=\left(\alpha_{0}, \alpha_{\infty}\right) \in \mathbb{R}^{2}$ and $1 \leq q \leq \infty$ such that

$$
\alpha_{0}+1 / q<0<\alpha_{\infty}+1 / q \text {. }
$$

Then, for any Banach couple $\left(A_{0}, A_{1}\right)$, we have with equivalence of norms $\left(A_{0}, A_{1}\right)_{1, q, \mathbb{A}}=\left(A_{0}, A_{1}\right)_{1, q, \mathbb{A}+1}^{J}$.

We finish the paper with the case $0<q \leq p \leq 1$ where we have also equality.

Theorem 4.10. Let $0<q \leq p \leq 1$ and $\mathbb{A}=\left(\alpha_{0}, \alpha_{\infty}\right) \in \mathbb{R}^{2}$ satisfying $\alpha_{0}+1 / q<$ $0 \leq \alpha_{\infty}+1 / q$. Then, for any $p$-Banach couple $\bar{A}=\left(A_{0}, A_{1}\right)$, we have with equivalence of quasi-norms

i) $\left(A_{0}, A_{1}\right)_{1, q, \mathbb{A}}=\left(A_{0}^{\sim}, A_{1}^{\sim}\right)_{1, q, \mathbb{A}+1 / q}^{J}$ if $\alpha_{\infty}+1 / q>0$,

ii) $\left(A_{0}, A_{1}\right)_{1, q, \mathbb{A}}=\left(A_{0}^{\sim}, A_{1}^{\sim}\right)_{1, q, \mathbb{A}+1 / q,(0,1 / q)}^{J}$ if $\alpha_{\infty}+1 / q=0$.

Proof. Recall that $\left(A_{0}, A_{1}\right)_{1, q, \mathbb{A}}=\left(A_{0}^{\sim}, A_{1}^{\sim}\right)_{1, q, \mathbb{A}}$. Applying (3.3), using that $q \leq p$ and Lemma 3.4 we get that

$$
\left(A_{0}, A_{1}\right)_{1, q, \mathbb{A}} \hookleftarrow \begin{cases}\left(A_{0}^{\sim}, A_{1}^{\sim}\right)_{1, q, \mathbb{A}+1 / q}^{J} & \text { if } \alpha_{\infty}+1 / q>0 \\ \left(A_{0}^{\sim}, A_{1}^{\sim}\right)_{1, q, \mathbb{A}+1 / q,(0,1 / q)}^{J} & \text { if } \alpha_{\infty}+1 / q=0 .\end{cases}
$$

In order to check the converse embedding, consider the function $\mathrm{v}_{q, \mathbb{A}}(\cdot)$. By Lemma 2.2, we have

$$
\mathrm{v}_{q, \mathbb{A}}\left(2^{k}\right) \sim \begin{cases}2^{-k} \ell^{\mathbb{A}+1 / q}\left(2^{k}\right) & \text { if } \alpha_{\infty}+1 / q>0, \\ 2^{-k} \ell^{\mathbb{A}+1 / q}\left(2^{k}\right) \ell \ell^{(0,1 / q)}\left(2^{k}\right) & \text { if } \alpha_{\infty}+1 / q=0 .\end{cases}
$$

Take any $a \in\left(A_{0}^{\sim}, A_{1}^{\sim}\right)_{1, q, \mathbb{A}}$. Since $(2.2)$ holds, we know that $\left(A_{0}^{\sim}, A_{1}^{\sim}\right)_{1, q, \mathbb{A}} \subseteq$ $\left(A_{0}^{\sim}+A_{1}^{\sim}\right)^{\circ}$. Using [27, Theorem 3.2], we can find $\left(u_{m}\right) \subseteq A_{0}^{\sim} \cap A_{1}^{\sim}$ with $a=\sum_{m=-\infty}^{\infty} u_{m}$ in $A_{0}^{\sim}+A_{1}^{\sim}$ and

$$
\left(\sum_{m=-\infty}^{\infty}\left[\min \left(1,2^{k-m}\right) J\left(2^{m}, u_{m}\right)\right]^{q}\right)^{1 / q} \leq c K\left(2^{k}, a\right), k \in \mathbb{Z} .
$$

Therefore, if $\alpha_{\infty}+1 / q>0$, according to (4.15) and (4.16), we have

$$
\|a\|_{\left(A_{0}^{\sim}, A_{1}^{\sim}\right)_{1, q, \mathbb{A}+1 / q}^{J}} \leq\left\|\left(J\left(2^{m}, u_{m}\right)\right)\right\|_{\ell_{q}\left(2^{-m} \ell^{\mathbb{A}+1 / q}\left(2^{m}\right)\right)}
$$




$$
\begin{aligned}
& \sim\left(\sum_{m=-\infty}^{\infty} J\left(2^{m}, u_{m}\right)^{q} \sum_{k=-\infty}^{\infty}\left[\min \left(1,2^{k-m}\right) 2^{-k} \ell^{\mathbb{A}}\left(2^{k}\right)\right]^{q}\right)^{1 / q} \\
& \lesssim\left(\sum_{k=-\infty}^{\infty} 2^{-k q} \ell^{\mathbb{A} q}\left(2^{k}\right) K\left(2^{k}, a\right)^{q}\right)^{1 / q} \\
& =\|a\|_{\left(A_{0}, A_{1}^{\sim}\right)_{1, q, \mathbb{A}}} .
\end{aligned}
$$

The case $\alpha_{\infty}+1 / q=0$ can be treated analogously.

Writing down Theorem 4.10 for $\left(A_{0}, A_{1}\right)$ a Banach couple, we recover a previous result of the authors [5, Theorem 3.2].

Acknowledgement. The authors have been supported in part by MTM201784508-P (AEI/ FEDER, UE). B. F. Besoy has also been supported by FPU grant FPU16/02420 of the Spanish Ministerio de Educación, Cultura y Deporte.

The authors would like to thank the referee for his/her useful comments which have led to improve the paper.

\section{References}

[1] Bennett, G., Some elementary inequalities. Q. J. Math. 38 (1987), $401-425$.

[2] Bennett, G., Some elementary inequalities, III. Q. J. Math. 42 (1991), 149 174.

[3] Bennett, C. and Sharpley, R., Interpolation of Operators. Boston: Academic Press 1988.

[4] Bergh, J. and Löfström, J., Interpolation Spaces. An Introduction. Berlin: Springer 1976.

[5] Besoy, B.F. and Cobos, F., Duality for logarithmic interpolation spaces when $0<q<1$ and applications. J. Math. Anal. Appl. 466 (2018), $373-399$.

[6] Besoy, B.F. and Cobos, F., Logarithmic interpolation methods and measure of non-compactness. Quart. J. Math. 00 (2019) 1 - 23; doi:10.1093/qmathj/haz041.

[7] Besoy, B.F., Cobos, F. and Fernández-Cabrera, L.M., On the structure of a limit class of logarithmic interpolation spaces. Preprint, Madrid (2019).

[8] Brudnyǐ, Y. and Krugljak, N., Interpolation Functors and Interpolation Spaces Vol. 1. Amsterdam: North-Holland 1991.

[9] Butzer, P.L. and Berens, H., Semi-Groups of Operators and Approximation. Berlin: Springer 1967.

[10] Cobos, F. and Domínguez, O., On Besov spaces of logarithmic smoothness and Lipschitz spaces. J. Math. Anal. Appl. 425 (2015), $71-84$. 
[11] Cobos, F., Domínguez, O. and Triebel, H., Characterizations of logarithmic Besov spaces in terms of differences, Fourier-analytical decompositions, wavelets and semi-groups. J. Funct. Anal. 270 (2016), $4386-4425$.

[12] Cobos, F., Fernández-Cabrera, L.M., Kühn, T. and Ullrich, T., On an extreme class of real interpolation spaces. J. Funct. Anal. 256 (2009), 2321 - 2366.

[13] Cobos, F., Fernández-Cabrera, L.M. and Mastyło, M., Abstract limit J-spaces. J. Lond. Math. Soc. 82 (2010), $501-525$.

[14] Cobos, F. and Kühn, T., Equivalence of K- and J-methods for limiting real interpolation spaces. J. Funct. Anal. 261 (2011), 3696 - 3722.

[15] Cobos, F., Kühn, T. and Schonbek, T., One-sided compactness results for Aronszajn-Gagliardo functors. J. Funct. Anal. 106 (1992), $274-313$.

[16] Cobos, F. and Segurado, A., Description of logarithmic interpolation spaces by means of the J-functional and applications. J. Funct. Anal. 268 (2015), 2906 -2945 .

[17] Cwikel, M. and Peetre, J., Abstract K- and J-spaces. J. Math. Pures Appl. 60 (1981), $1-50$.

[18] Doktorskii, R.Y., Reiteration relations of the real interpolation method. Soviet Math. Dokl. 44 (1992), 665 - 669.

[19] Edmunds, D.E. and Evans W.D., Hardy Operators, Function Spaces and Embeddings. Berlin: Springer 2004.

[20] Edmunds D.E. and Opic B., Limiting variants of Krasnosel'skii's compact interpolation theorem. J. Funct. Anal. 266 (2014), 3265 - 3285.

[21] Evans, W.D. and Opic, B., Real interpolation with logarithmic functors and reiteration. Canad. J. Math. 52 (2000), 920 - 960.

[22] Evans, W.D., Opic, B. and Pick, L., Real interpolation with logarithmic functors. J. Inequal. Appl. 7 (2002), 187 - 269.

[23] Gustavsson, J., A function parameter in connection with interpolation of Banach spaces. Math. Scand. 42 (1978), 289 - 305.

[24] Janson, S., Minimal and maximal methods of interpolation. J. Funct. Anal. 44 (1981), $50-73$.

[25] König, H., Eigenvalue distributions of compact operators. Basel: Birkhäuser 1986.

[26] Köthe, G., Topological Vector Spaces I. Berlin: Springer 1969.

[27] Nilsson, P., Reiteration theorems for real interpolation and approximation spaces. Ann. Mat. Pura Appl. 132 (1982), 291 - 330.

[28] Opic, B. and Pick, L., On generalized Lorentz-Zygmund spaces. Math. Inequal. Appl. 2 (1999) $391-467$.

[29] Ovchinnikov, V.I., The Method of Orbits in Interpolation Theory. Math. Rep. 1 (1984), $349-515$.

[30] Peetre, J., A Theory of Interpolation of Normed Spaces. Notes Mat. 39. Brasilia: Lecture Notes 1963. 
[31] Persson, L-E., Interpolation with a parameter function. Math. Scand. 59 (1986), $199-222$.

[32] Triebel, H., Interpolation Theory, Function Spaces, Differential Operators. Amsterdam: North-Holland 1978.

Received 